Advances in High-Pressure Technology for Geophysical Applications

Jiahua Chen, Yanbin Wang, T.S. Duffy, Guoyin Shen, L.F. Dobrzhinetskaya, editors

(C) 2005 Elsevier B.V. All rights reserved.

\title{
Chapter 4 \\ Simultaneous determination of elastic and structural properties under simulated mantle conditions using multi-anvil device MAX80
}

\author{
Hans J. Mueller, Christian Lathe and Frank R. Schilling
}

\begin{abstract}
An ultrasonic interferometry high-pressure set-up for elastic wave velocity measurements under simulated Earth's mantle conditions has been developed. A DIA-type multi-anvil apparatus MAX80 permanently located at HASYLAB, Hamburg, Germany for X-ray diffraction (XRD) under in situ condition was equipped for simultaneous ultrasonic measurements. Two of the six anvils were equipped with lithium niobate $P$ - and $S$-wave transducers of $33.3 \mathrm{MHz}$ natural frequency. The pressure and temperature limits of the high-pressure apparatus were not reduced as a side effect of the modification. The ultrasonic configuration allows all kinds of interferometric measurements with compressional and shear waves. In addition to the classical ultrasonic interferometry the newly developed data transfer function technique (DTF), first described in [J. Phys. Condens. Matter 14 (2002) 11337], is discussed in detail to give the readers the chance to use this valuable and important new method.

The results for natural San Carlos olivine up to 3 GPa pressure are compared with published data of several authors. The data for hot-isostatic-pressed anorthite solved discrepancies between published high-pressure and normal-pressure data for polycrystalline anorthite leading to $v_{p}=7.28 \mathrm{~km} / \mathrm{s}$, $v_{s}=3.93 \mathrm{~km} / \mathrm{s}$ at ambient conditions and $d v_{p} / d p=0.027 \mathrm{~km} / \mathrm{s} \mathrm{GPa}, d v_{s} / d p=0.001 \mathrm{~km} / \mathrm{s} \mathrm{GPa}$. The obtained data sets correspond to published results within the accuracy of the method.

As an example for unquenchable phase transitions we measured the elastic wave velocities at the high-pressure clinoenstatite $\left(\mathrm{MgSiO}_{3}, \mathrm{HCEn}\right)$ - low-pressure (LCEn) transition at high pressure, high temperature conditions in conjunction with in situ XRD. For ultrasonic interferometry experiments LCEn powder synthesized at ambient pressure was hot-isostatic-pressed at 0.4 GPa and $1400^{\circ} \mathrm{C}$ for $2 \mathrm{~h}$ in MAX80 to obtain low-porosity samples. The elastic wave velocities $v_{p}$ and $v_{s}$ of the CEn sample were measured in situ using the classical interferometric technique as well as the recently developed ultrasonic data transfer function (DTF) technique for the elastic wave velocities as a function of pressure at $700^{\circ} \mathrm{C}$. To compare the results, $v_{p}$ and $v_{s}$ were measured at 6.7 and $7.5 \mathrm{GPa}$ using both interferometric techniques. The results correspond within the limits of less than $1 \%$.
\end{abstract}

\section{Introduction}

During the last decade the progress of global seismology in general and of the tomographic method in particular in terms of resolution, amount of data, and quality of their processing reveals a lot of new and exciting structural details of the Earth's deep interior (van der Hilst, 1995; Li et al., 2000). Understanding and modeling of mantle dynamics, crust mantle interaction, formation of plumes, and many others require much more detailed insights into the structural and physical properties of materials relevant for great depths 
(Kohlstedt et al., 1996). Ultradeep subduction, penetrating into the lower mantle, the incidence of deep earthquakes and their relation to the nature of the transition zone as well as the discussion of slab recycling in plumes require comparable effort in high-pressure research and precise geophysical observation. Different multi-anvil high-pressure devices have been used with great success in experimental mantle mineralogy for many years (Shimomura et al., 1985; Yagi, 1988; Funamori et al., 1996a,b; Suzuki et al., 1996; Oguri et al., 2000; Hirose et al., 2001; Nishiyama and Yagi, 2003). In many cases pioneering work was achieved at the Mineral Physics Institute, Stony Brook, e.g. ultrasonic interferometry in the DIA-type multi-anvil cell SAM85 (Chen et al., 1996; Li et al., 1996a,b, 2001a, 1998; Kung and Rigden, 1999; Kung et al., 2000, 2001a,b, 2002; Li, 2003), development of the DTF technique (Li et al., 2002), X-radiography (Li et al., 2001b), and double-stage T-CUP up to $23 \mathrm{GPa}$ for in situ X-ray diffraction (XRD) (Vaughan, 1993; Vaughan et al., 1995). The in situ study of complex phase relations, the understanding and description of unquenchable high-pressure phases, and the investigation of the kinetics of phase transitions and mineral reactions require time-resolved measurements. Synchrotron radiation allows in situ structural investigations under simulated mantle conditions. Furthermore, simultaneous measurement of compressional and shear wave velocities, especially using the DTF technique, and structural investigation might be the critical key to understand the ongoing processes in more detail.

The different elastic properties, elastic wave velocities, shear modulus, bulk modulus, Young's modulus, Poisson's ratio, provide detailed information about the mechanical behavior of samples (Kern, 1982). Elastic properties are particularly sensitive to phase transitions. The existing knowledge of the physical properties of high-pressure phases is mostly limited to equilibrium conditions. Considering the significance of non-equilibrium structures for the Earth's deep interior, reliable time-resolved measurements during transition processes are required. Simultaneously determined elastic wave velocities and structural information provides an independent way to measure compressibility and elastic moduli without pressure calibrant, independent of any standard material (Spetzler and Yoneda, 1993; Yoneda and Spetzler, 1994; Getting, 1998; Zha et al., 2000; Mueller et al., Q1 2002) (see also Mueller et al., pp. xxx, this volume). Elastic properties are important for thermodynamic calculations and to understand the kinetics of mineral reactions (Haussuehl et al., 1980; Hoffbauer et al., 1985; Lauterjung and Will, 1985; Angel and Ross, 1996; Zinn et al., 1997).

In addition to the description of up-to-date ultrasonic interferometric techniques we present exemplary results of high-pressure interferometric measurements of compressional and shear wave velocities in single-crystal San Carlos olivine polycrystalline anorthite, and at the high- $P$ (HCEn)-low- $P$ (LCEn) clinoenstatite transition (Mueller Q2 et al., 2002, 2003, 2004).

Enstatite, the pure magnesium silicate end-member of pyroxene stoichiometry, $\mathrm{MgSiO}_{3}$, exists in at least five polymorphs (Bowen and Andersen, 1914). Using singlecrystal XRD analyses in a diamond anvil cell, Angle et al. (1992) determined the clinoenstatite (CEn) transformation from the $P 2_{1} / c$-to the $C 2 / c$-polymorph to be at about 5.5-8.0 GPa and room temperature (RT) conditions. They also determined the structure of the HCEn polymorph and estimated thermodynamic data for the CEn-transition. A further important conclusion of their study was that the LCEn-HCEn transition is not quenchable, reverting to the $P 2_{1} / c$-structure upon decompression at RT. The current 
understanding of phase relations in the system $\mathrm{MgSiO}_{3}$ is summarized, e.g. by Presnell Q3 (1995, Fig. 8). Up to now, the position of the $\mathrm{HCEn}-\mathrm{LCEn}$ transition for $\mathrm{MgSiO}_{3}$ is only deduced from thermodynamic data by Angel and Hugh-Jones (1994). Additionally, large discrepancies exist between recently performed experimental studies for the OEn-HCEn transition at high pressures and temperatures. For clarification of these discrepancies and to better characterize the HCEn-LCEn transition we performed in situ experiments at elevated temperatures and various pressures.

\section{Techniques, methods and materials description}

\subsection{Multi-anvil high-pressure apparatus MAX80}

MAX80 is a DIA-type multi-anvil high-pressure apparatus with six tungsten carbide anvils compressing a cubic sample volume of maximum $8 \times 8 \times 8 \mathrm{~mm}^{3}$ (Fig. 1). The apparatus is installed at beamline F2.1 at the HamburgerSynchrotronstrahlungsLabor (HASYLAB) for high-pressure-high-temperature synthesis and in situ measurements. The anvils are driven by a $2500 \mathrm{~N}$ uniaxial hydraulic ram. Three anvil sets with different truncations exist $-6,5$, and $3.5 \mathrm{~mm}$. The corresponding cube length is 8,6 , and $5.5 \mathrm{~mm}$ resulting in maximum pressures of about 7,9 , and $12 \mathrm{GPa}$. The maximum attainable temperature is $2000 \mathrm{~K}$ produced by an internal graphite heater. One or two of the original anvil spacers had to be replaced by redesigned parts. The new spacers have a cavity in their center to keep the ultrasonic transducer free of stress from the load of the hydraulic press. The ultrasonic anvils are equipped with two P-wave and two S-wave transducers. Because of their high conversion factor and high thermal stability lithium niobate transducers overtone polished with a resonant frequency of $33.3 \mathrm{MHz}$ were cemented on the polished rear side of the ultrasonic anvils using epoxy resin diluted by acetone to reduce its viscosity for a minimum thickness of the glue film. The resulting strong coupling of the transducer to the anvil is of fundamental importance for the interferometric method, because the strong coupling results in a broad band characteristics of the transducer.

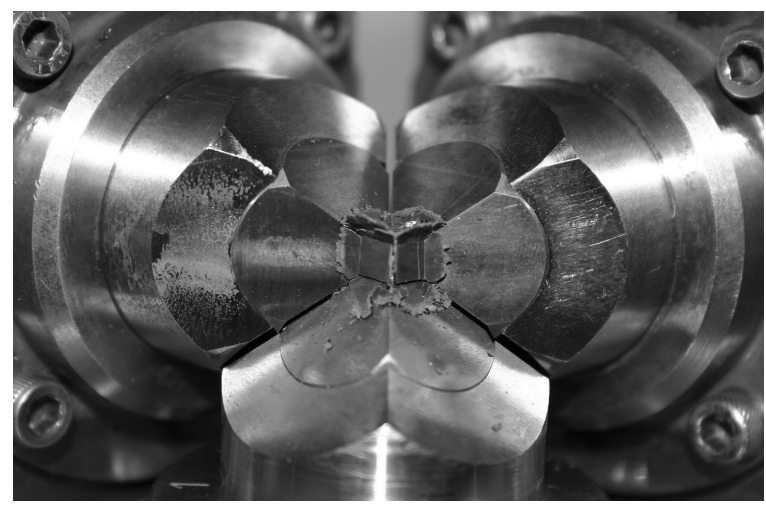

Figure 1. Boron-epoxy cube of MAX80 with gaskets after the experiment. Top and front lateral anvils (6 $\mathrm{mm}$ truncation) are removed. 
Diffraction patterns are recorded in an energy-dispersive mode using X-rays from the storage ring DORIS III at HASYLAB and a $\mathrm{Ge}$-detector. $\mathrm{NaCl}$ is used as pressure standard. The pressure is calculated using an in-house program from the XRD data following the EoS of Decker (1971). For further details, see Mueller et al., pp. xxx, this Q1 volume and Mueller et al. (2002, 2003).

\subsection{Multi-anvil apparatus cell assembly}

The high-pressure cell consists of a cube made by pressing and adjacent machining of epoxy resin mixed with amorphous boron with the weight ratio 1:4 for better compressive strength containing the ultrasonic configuration, the heater, the pressure standard, and the thermocouple (Fig. 2). The interfaces between the sample and the close-fitting buffer rods/ reflector bars are polished for optimal ultrasonic coupling. The sample is surrounded by a boron nitride sleeve for electrical insulation and as pressure transmitting medium inside a stepped graphite heater. The stepping results in stronger heat production at the ends of the sample which compensates the heat flow to the colder anvils resulting in a smaller temperature gradient throughout the sample (Knoche et al., 1997, 1998). For experiments, if sodium chloride is not in use as ultrasonic reflector, a sleeve made from a mixture of sodium chloride and boron nitride is used as pressure calibrant. The copper rings contact the heater at the top and bottom anvils, and the pyrophyllite rings are a quasi-hydrostatic pressure transmitting medium. The total length of the set-up (see Fig. 2) reduced from $8 \mathrm{~mm}$ to about $6.9 \mathrm{~mm}$ during the experiments by plastic deformation. Even very brittle buffer rods made from fused quartz or polycrystalline corundum did not crush during the

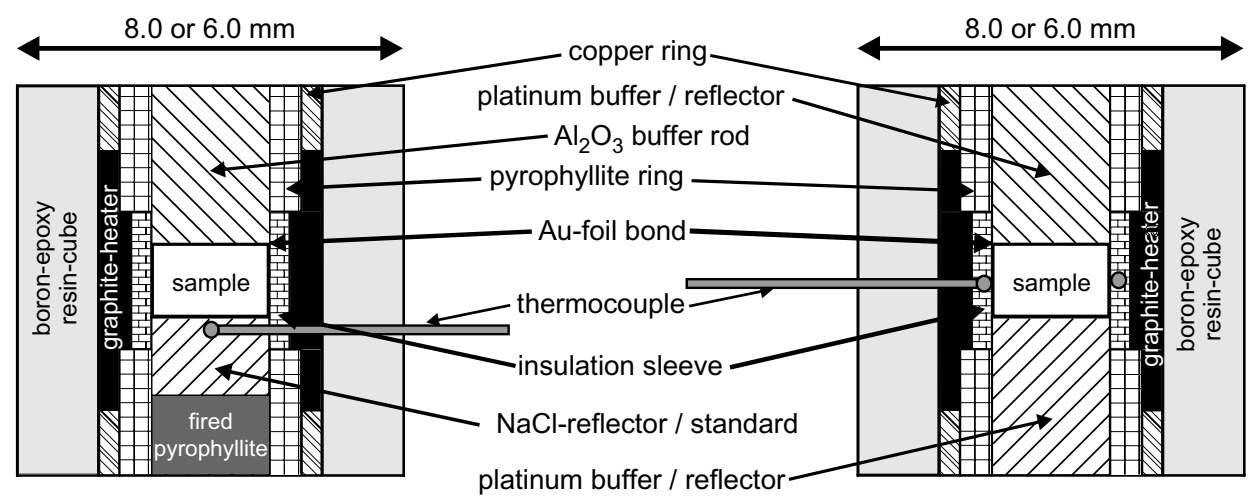

Figure 2. MAX80 set-ups for combined XRD and ultrasonic measurements. For a low-loss transmission of ultrasonic energy from the buffer rod made of brittle material to the sample the interface is covered by $\mathrm{Au}$ foils. The $\mathrm{NaCl}$ cylinder reflects the ultrasonic waves back to the transducer and is used as pressure standard at the same time. The adjacent fired pyrophyllite part prevents the blow-out of the $\mathrm{NaCl}$ cylinder at elevated temperatures. Opposite to set-ups for only XRD measurements the thermocouple has to be located outside the sample center to keep the ultrasonic travel path undisturbed. The sample is surrounded by a hBN-sleeve and the adjacent graphite heater. The copper rings contact the heater to the top and bottom tungsten carbide anvils. The boron-epoxy resin cube is a pressure transmitting medium highly permeable for X-rays. 
experiments. Olivine and anorthite only deformed elastically as measured by a dial gauge indicator before and after the experiments. Corresponding to the anvil sets with truncations of 6,5 , and $3.5 \mathrm{~mm}$ cell assemblies with 8,6 , and $5 \mathrm{~mm}$ length of the sides were designed. A thermocouple inside the graphite heater on the sample surface, or inside the $\mathrm{NaCl}$ reflector close to the sample is used to control the temperature. The temperature inside the sample at its center is derived from a calibration using special cell assemblies with an additional thermocouple at the sample center or inside the $\mathrm{NaCl}$ reflector and a calibration of electrical current, respectively. The maximum deviation of the temperature measured during the experiments from the true sample temperature was found to be $10-25^{\circ}$ depending on the method and on cube deformation. To minimize electromagnetically induced noise to the ultrasonic signal, a DC power source was used for heating. Even DC electrical heating requires the grounding of the anvil where the transducers are assembled to avoid interferences of the electric current with the transducers during heating.

A stress test was performed to get quantitative information about the level of nonhydrostatic stress inside the sample at cold compression, especially. A common way to do this is measuring the unit cell deformation of $\mathrm{NaCl}$. Over all pressure conditions up to $8 \mathrm{GPa}$ and the unit cell parameters derived from 111 to 200 were compared. We found maximum deviation of the calculated volumes of the unit cell between +0.03 and +0.25 , i.e. any differential stress resulting in negative quotients were not found. As an additional indication for a high degree of hydrostatic pressure conditions at different elevated temperatures we found no shift of the phase boundary between high- $P$ (HCEn)-low-P (LCEn) clinoenstatite (see Section 3.3) derived from the results of experiments using a powder or a hot-isostatic-pressed (HIP) sample, otherwise the last-mentioned samples should apparently cross the phase boundary at lower pressure conditions because of the higher internal stress. In this case one or two of the unit cell parameters (see Table 1 and Fig. 17) would also systematically deviate from

Table 1. Variation of CEn unit cell parameters with pressure, and temperature.

\begin{tabular}{lrlllll}
\hline$P(\mathrm{GPa})$ & $T\left({ }^{\circ} \mathrm{C}\right)$ & $a(\AA)$ & $b(\AA)$ & $c(\AA)$ & $\beta(-)$ & $V\left(\AA^{3}\right)$ \\
\hline LCEn & & & & & & \\
$6.61(5)$ & 300 & $9.438(18)$ & $8.624(12)$ & $5.076(9)$ & $107.5(2)$ & $394.1(9)$ \\
$7.20(5)$ & 550 & $9.438(8)$ & $8.621(6)$ & $5.076(4)$ & $107.68(8)$ & $393.5(4)$ \\
$7.50(5)$ & 700 & $9.452(9)$ & $8.613(6)$ & $5.069(4)$ & $107.80(8)$ & $392.8(4)$ \\
$7.88(5)$ & 900 & $9.405(9)$ & $8.609(6)$ & $5.066(4)$ & $107.78(9)$ & $390.6(4)$ \\
HCEn & & & & & & \\
$6.61(5)$ & 20 & $9.224(9)$ & $8.658(6)$ & $4.915(4)$ & $101.71(7)$ & $384.4(4)$ \\
$6.61(5)$ & 250 & $9.244(8)$ & $8.665(4)$ & $4.925(5)$ & $101.54(5)$ & $386.5(9)$ \\
$7.20(5)$ & 34 & $9.195(8)$ & $8.623(6)$ & $4.919(4)$ & $101.63(7)$ & $382.0(4)$ \\
$7.20(5)$ & 500 & $9.239(5)$ & $8.671(4)$ & $4.927(3)$ & $101.56(6)$ & $386.7(4)$ \\
$7.50(5)$ & 41 & $9.197(8)$ & $8.615(6)$ & $4.906(4)$ & $101.49(8)$ & $380.9(4)$ \\
$7.50(5)$ & 650 & $9.237(9)$ & $8.643(5)$ & $4.925(3)$ & $101.68(5)$ & $385.0(9)$ \\
$7.88(5)$ & 20 & $9.188(10)$ & $8.593(7)$ & $4.896(5)$ & $101.71(9)$ & $378.5(5)$ \\
$7.88(5)$ & 850 & $9.222(5)$ & $8.651(6)$ & $4.910(5)$ & $101.52(4)$ & $383.8(9)$
\end{tabular}

The $1 \sigma$ uncertainties of the last digits of the lattice refinements are given in parentheses. 
published data, and from results of single-crystal DAC experiments, especially. All that was not found.

\subsection{Ultrasonic interferometry techniques}

\subsubsection{Frequency sweep method}

Ultrasonic interferometry, using the interference between the incident and reflected waves inside the sample, was first described by McSkimin (1950). It allows high-precision measurements of the travel time through the sample, independent of the delay travel time and its variation with pressure and temperature in anvils and buffer rods. Piezoelectric transducers for the generation and detection of ultrasonic waves are cemented at the rear Q1 side of the piston outside the true pressure cell (see Mueller et al., pp. xxx, this volume). The amount of energy reflected or transmitted at an interface is given by the reflection factor $R$, where

$$
R=\frac{Z_{2}-Z_{1}}{Z_{2}+Z_{1}}
$$

and

$$
Z=\rho v_{i}
$$

Q9 with $Z$ the acoustic impedance, $\rho$ density, $v_{i}$ the compressional or shear wave velocity and the transmission factor $D$

$$
D=\frac{2 Z_{2}}{Z_{2}+Z_{1}}
$$

with $Z_{1}$ the acoustic impedance of medium 1 and $Z_{2}$ the acoustic impedance of medium 2 .

For negative $R$ values a phase shift of $180^{\circ}$ is observed (see Niesler and Jackson, 1989). For example, if we take an olivine sample $\left(v_{\mathrm{p}} \approx 8.25 \mathrm{~km} / \mathrm{s}, \rho \approx 3.34 \mathrm{~g} / \mathrm{cm}^{3}\right)$ between a Q9 fused quartz buffer $\left(v_{\mathrm{p}} \approx 5.57 \mathrm{~km} / \mathrm{s}, \rho \approx 2.60 \mathrm{~g} / \mathrm{cm}^{3}\right)$ and a reflector made of platinum Q9 $\left(v_{\mathrm{p}} \approx 3.96 \mathrm{~km} / \mathrm{s}, \rho \approx 21.40 \mathrm{~g} / \mathrm{cm}^{3}\right)$, the reflection factor at the interface buffer-sample becomes $\approx 0.3$, and $>0.5$ at the transition to the reflector. This means that nearly three quarters of the energy reach the sample and half of the energy is reflected at the sample's rear side (Mueller et al., 2002).

The most popular interferometric technique is called double-pulse phase-comparison method (McSkimin, 1950). It effectively eliminates all interferences, which are not useful for further evaluation. Its precision is about 1-3 times higher (Schreiber et al., 1973; Li et al., 1998) than classical travel-time methods (Birch, 1960, 1961). The difference between several destructive and constructive interferences is used to reveal the reverberation time inside the sample. The high precision of the ultrasonic interferometry requires a highly precise sample length measurement under in situ conditions, because calculating the elastic wave velocities from the reverberation time requires the sample Q1 length (see Mueller et al., pp. xxx, this volume). Using a broad range of frequencies leads to the detection of a high number of constructive and destructive interferences, yielding to higher precision of the regression analysis (see Fig. 3). We mostly used a slightly modified 


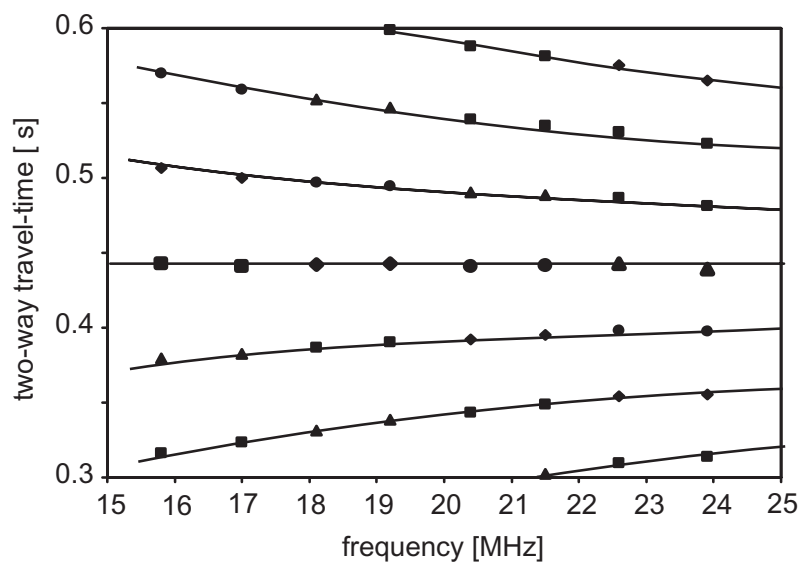

Figure 3. Travel time curves as a function of frequency. Picking all available maxima and minima as a function of frequency allows the determination of the travel time inside the sample as a regression result for the horizontal point sequence between the curves of opposite curvature.

method, similar to that published by Shen et al. (1998) for diamond anvil cells, where only one single tone burst (a sinusoidal wave limited in time to few microseconds) of about three to sixfold duration of the travel time inside the sample is used. The narrower frequency band of the prolonged burst increases the sharpness of the interference pattern compared to the shorter bursts of the double-pulse method. Because of the broader frequency range most of our measurements were performed using tone bursts of $4 \mu \mathrm{s}$ duration. Additional coupling media, e.g. gold foil as described by Niesler and Jackson (1989), are only necessary at the interface between two brittle media, e.g. corundum buffer rod and San Carlos olivine sample or for making the evaluation of X-radiograms (see Q1 Mueller et al., pp. xxx, this volume) easier (Li et al., 2001a,b).

Ultrasonic interferometry requires a special equipment for generation, superposition, and display of rf-signals. In the last two decades of the 19th century, the Australian Scientific Instruments Ultrasonic Interferometer (Rigden et al., 1988, 1992; Niesler and Jackson, 1989), became the standard equipment. Today a combination of digital generators and oscilloscopes controlled by a PC took on the task. For details of the Q1 electronic equipment used for our measurements see Mueller et al., pp. xxx, this volume.

\subsubsection{Ultrasonic data transfer function technique}

\subsubsection{Pulse shaping of the excitation function}

The classical digital sweep interferometry is very time consuming. A $60 \mathrm{MHz}$ frequency sweep with $100 \mathrm{kHz}$ steps lasts for more than $30 \mathrm{~min}$. Consequently a single measurement of $v_{\mathrm{p}}$ and $v_{\mathrm{s}}$ requires more than $1 \mathrm{~h}$. This is a serious limitation not only for all transient measurements, but also limits the data collection at elevated temperatures to prevent the boron-epoxy cubes and the anvils from overheating. So the ultrasonic interferometry is the limiting factor for the experiments. The measurement can be made faster by limiting the frequency sweep to few $\mathrm{MHz}$ and increasing the frequency steps to $200 \mathrm{kHz}$ or more. 
But this limits the accuracy of the method significantly (see Section 2.3.1). A solution is the generation and emission of all the single "monochromatic" frequencies simultaneously. This was first described by Li et al. (2002), the ultrasonic DTF technique. Based on discussions with B. Li, and initiated by him, the technique, described here was developed independently at GFZ (GeoForschungsZentrum Potsdam), Dept. 4.

At first we will look how a digital sweep measurement is performed practically. The generator has the data for all the monochromatic frequency waves in the desired bandwidth with a given step rate as files in its memory. By PC-command the files were called-in and the waves were generated one after another. An oscilloscope receives and digitizes the resulting signals and saves them at the hard drive, also step by step. If we plan to "unify" these consecutive actions, it is obvious to create an excitation function as the sum of all these already existing files for the whole frequency range. Exactly that we did as our first approach.

$$
Y(t)=\sum_{i=1}^{i=n} y_{i}(t)
$$

with $Y$ the amplitude of summarized excitation function, $y_{t}$ the amplitude of sinusoidal Q4 waves between upper and lower cut-off frequency and $t$ time (Fig. 4).

Figure $8 \mathrm{a}$ and $\mathrm{b}$ shows the result for the whole $4 \mu$ s duration and the first $200 \mathrm{~ns}$ with higher time magnification. The function increases very steeply from zero followed by a little less dramatic decrease and a relatively low attenuation for later points in time. If we want to apply this to an ultrasonic transducer, we have to realize that first of all the transducer is a mechanical vibratory system driven by piezoelectricity and its reversal, respectively, i.e. we have to keep in mind its inertia. The excitation function would act as a shock. The transducer would mostly respond with an attenuated oscillation in its natural frequency. The excitation must be much more intensive to make the forced oscillations of the transducer stronger, far from resonance. What we have to do is inverting the function in time and amplitude, removing the first point - 0 - and "assembling" this inverted function to the beginning of the original function. Now we have a slowly increasing oscillation culminating in two consecutive, but opposite symmetrical deflections followed by a slow tailing off. Because the function has two times the maximum duration of the used arbitrary
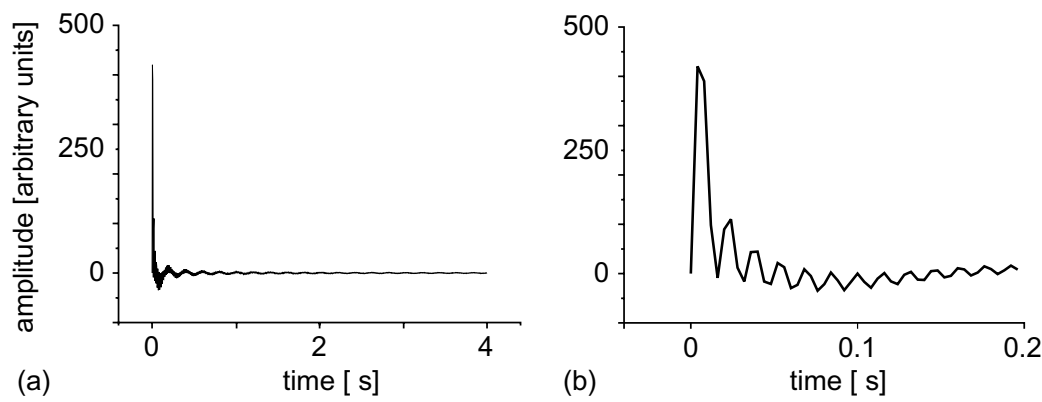

Figure 4. Excitation function (sine-pulse) for ultrasonic data transfer function technique calculated by summation of sinusoidal waves of $4 \mu$ s duration from 5 to $65 \mathrm{MHz}$ with a step rate of $100 \mathrm{kHz}$, (a) time base $4 \mu \mathrm{s}$, (b) time base $200 \mathrm{~ns}$. 


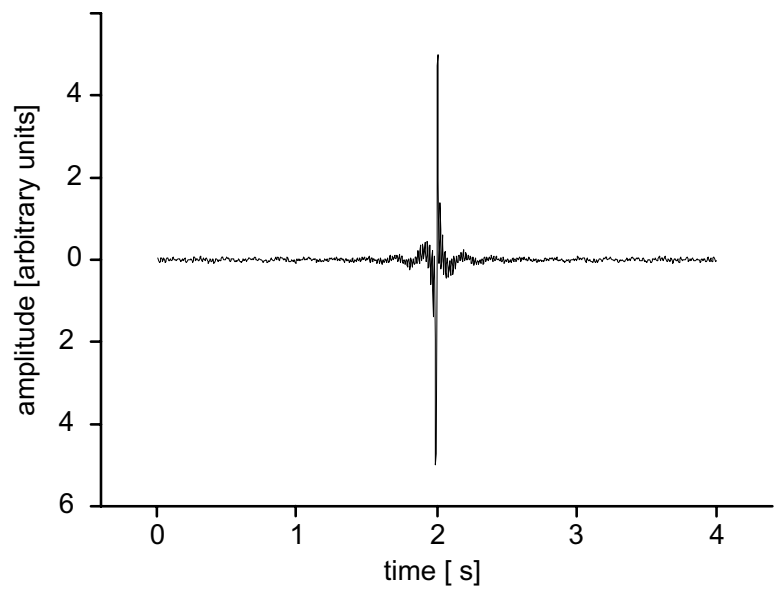

Figure 5. Completed sine-pulse excitation function created from the sine-pulse (Fig. 4) by "assembling" its copied function inverted in time and amplitude at the beginning of the original function.

waveform generator we have to cut-off the first and last $2 \mu \mathrm{s}$. This results in limited deformation of the frequency spectrum (Seidel and Myszkowski, 2004), but it is much simpler than the other option writing a sequence file, i.e. a command for using more than one file after another, because in this case we have to check very carefully that the switching time among the files is much less than the sampling rate. Otherwise we have to remove the corresponding number of points, because the function is only very effective, if it is totally symmetrical in amplitude and time. Actually this sine-pulse (Fig. 5) was very successfully used in many of our experiments. If we look to high-frequency engineering, pulses of this type are rarely used in spite of their effective excitation, because of their high demands on symmetry. Otherwise the spectrum deforms dramatically. This disadvantage can be limited by using a cosine-pulse created by adding up all single waves starting each of them with phase $\pi / 2$, i.e. the cosine function. Figure 6 shows the result in two different time bases, analogous to Figure 8. Comparing both indicates the dying down seems to be a
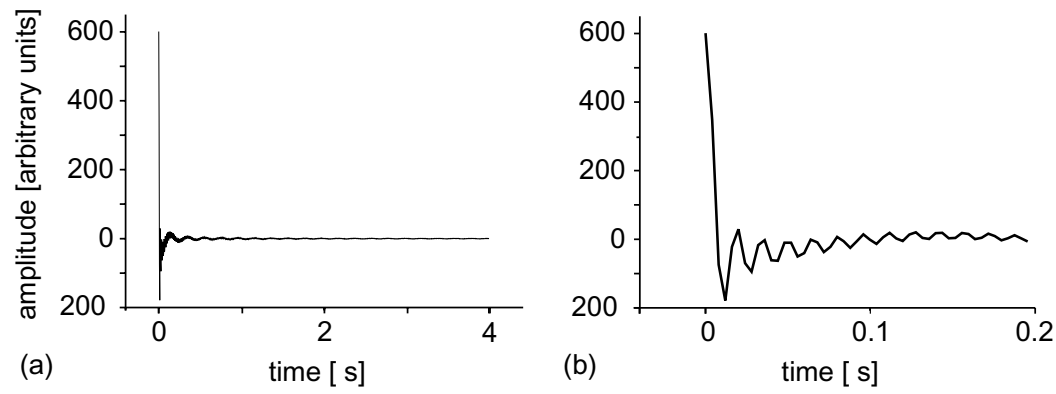

Figure 6. Cosine-pulse excitation function for ultrasonic data transfer function technique calculated by summation of cosinusoidal waves of $4 \mu$ s duration from 5 to $65 \mathrm{MHz}$ with a step rate of $100 \mathrm{kHz}$, (a) time base $4 \mu \mathrm{s}$, (b) time base $200 \mathrm{~ns}$. The angularity (see b) is a result of the limited resolution in time and of the discrete frequency spectrum, especially. 
little faster for the cosine-function. Both excitation functions work very well and are simple to calculate by each user. Moreover, because of its creation as the sum of single wave files, it is also very simple to suppress transducers resonance very effectively, even individually for each oscillating system, by multiplying each single file with a factor corresponding to the inverted, measured resonance curve of the transducer-glue anvil system.

If we look in detail to our sine-pulse and cosine-pulse functions (Figs. 4 and 6) we find they look multi-cornered, non-smooth. This is the result of the limited sampling rate of $250 \mathrm{MHz}$ and the frequency step of $100 \mathrm{kHz}$, i.e. both our functions do not represent a continuous frequency spectrum between both cut-off frequencies. The problem is very well investigated, because it plays an important role in up-to-date communication technology. High-speed data transmission using channels of limited bandwidth (cell phones, modems, digital TV, digital cameras, camcorders, etc.) without inter-symbol interference (ISI) require thorough pulse optimization. So, a channel specified by pulse response $h(t)$ is ISI free, if

$$
H\left(\mathrm{e}^{-\mathrm{j} 2 \pi f T}\right)=\frac{1}{T} \sum_{n=-\infty}^{\infty} H\left(f+\frac{n}{T}\right)=1
$$

This condition is called Nyquist Criterion. We will come back to this at the end of the section. A $h(t)$ that satisfies Nyquist criterion is called Nyquist pulse (Ekbal, 2004). The transfer function $H(\omega)$ of the ideal Nyquist filter is rectangular with single-sided bandwidth $\omega_{0}$ :

$$
H(\omega) / T \begin{cases}1 & \text { for }-1<\omega / \omega_{0}<1 \\ 0 & \text { otherwise }\end{cases}
$$

or

$$
H(\omega) \begin{cases}T & \text { for }-17(2 T)<\omega /(2 \pi)<1 /(2 T) \\ 0 & \text { otherwise }\end{cases}
$$

The impulse response (inverse Fourier transform; of $H$ ) is then

$$
h(t)=\sin c\left(\frac{t}{T}\right) \equiv \frac{\sin \left(\pi \frac{t}{T}\right)}{\left(\pi \frac{t}{T}\right)}
$$

This is the Nyquist pulse with minimum bandwidth (Chan, 2004). The Nyquist pulse, displayed for a cut-off frequency of $65 \mathrm{MHz}$ (see Fig. 7), corresponds to our abovecalculated cosine-pulse, but with a continuous frequency spectrum inside the bandwidth. Consequently it also dies out very slowly, and any cut-off results in non-flat parts in spatial domain, i.e. uncontrolled non-uniform amplitudes at different frequencies. The solution is the family of raised-cosine pulses (Seidel and Myszkowski, 2004). The function of the 


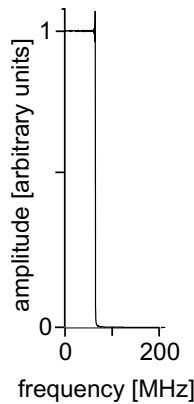

(a)

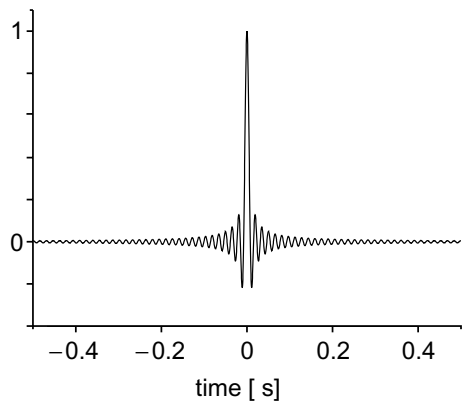

(b)

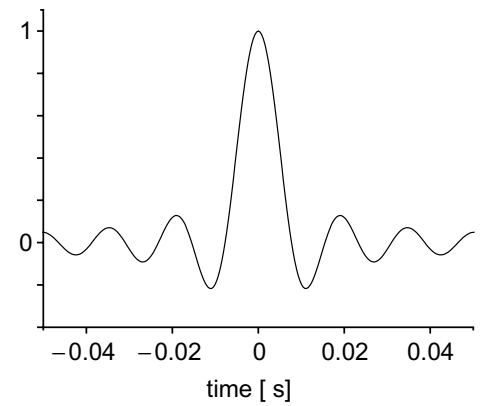

(c)

Figure 7. Sine-function for a low-pass filter with a cut-off frequency of $65 \mathrm{MHz}$, displayed in two time bases including its FFT; (a) FFT, (b) time base -0.5 to $0.5 \mu \mathrm{s}$, (c) time base -0.05 to $0.05 \mu \mathrm{s}$.

ideal Nyquist pulse $-\sin c(t / T)-$ is multiplied by an additional fall off function.

$$
h(t)=\sin c\left(\frac{t}{T}\right)\left[\frac{\cos \left(\frac{\alpha \pi t}{T}\right)}{1-\left(\frac{2 \alpha \pi t}{T}\right)^{2}}\right]
$$

with $\alpha$ the roll-off factor controlling the slope steepness of frequency spectrum function, $0<\alpha<1$.

The raised cosine-pulse falls as $1 / \alpha^{2} t^{3}$, whereas the ideal Nyquist pulse only falls as $1 / t$. The raised-cosine transfer function is the corresponding Fourier transform.

$$
\begin{array}{ccc}
T & |\omega| \leq(1-\alpha) \frac{\pi}{T} \\
H(\omega)=\frac{T}{2}\left[1-\sin \left(\frac{T}{2 \alpha}\left(|\omega|-\frac{\pi}{T}\right)\right)\right] & (1-\alpha) \frac{\pi}{T} \leq|\omega| \leq(1+\alpha) \frac{\pi}{T} \\
0 & (1+\alpha) \frac{\pi}{T} \leq|\omega|
\end{array}
$$

Figure 8 compares the Nyquist pulse and the raised-cosine pulse with different roll-off factors $\alpha$ in time and spatial domain (modified from Rapppaport, 2002; Chan, 2004). To complete things it should be mentioned that already further developed pulses exist as square-root raised-cosine pulse, the "better than" Nyquist-pulse (Beaulieu et al., 2001) and others. But our demands for the DTF technique are met by the raised-cosine pulse with one exception - the resonance suppression. To implement this for the available pulses we will use the fast Fourier transform (FFT), a special form of the discrete Fourier transform, available at many PC-software and installed at many digital oscilloscopes. The relation (Ekbal, 2004) between discrete-time Fourier transform $P\left(\mathrm{e}^{-\mathrm{j} 2 \pi f T}\right)$ and continuous-time Fourier transform $P(f)$ is

$$
P\left(\mathrm{e}^{-\mathrm{j} 2 \pi f T}\right)=\frac{1}{T} \sum_{n=-\infty}^{\infty} P\left(f+\frac{n}{T}\right)
$$




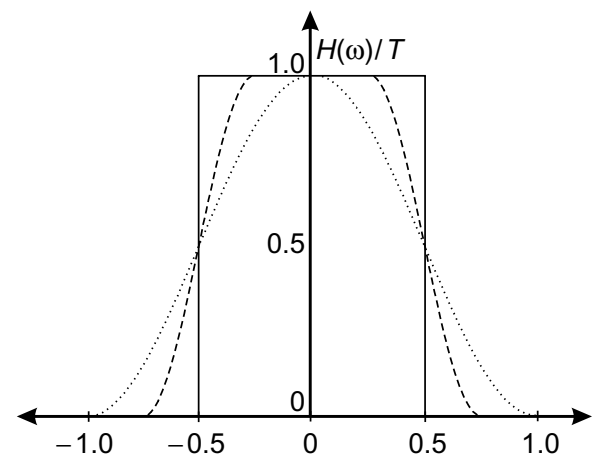

(a)

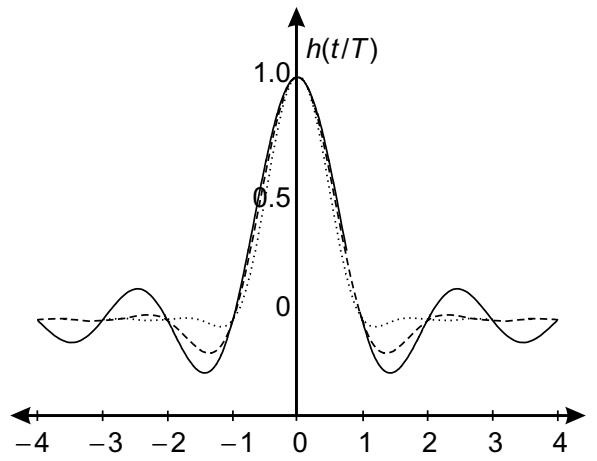

(b)

Figure 8. Comparison of transfer functions $H(\omega)$ of the ideal Nyquist pulse and the raised-cosine pulse with different roll-off factors, as well as their impulse responses (inverse Fourier transform of $H$ ) in spatial domain. A raised cosine-pulse with roll-off factor $\alpha=0$ is an ideal Nyquist pulse (modified from Chan, 2004): - $\alpha=0 ;---, \alpha=0.5 ; \cdots . ., \alpha=1$.

We use our low-pass cosine-pulse with a cut-off frequency of $65 \mathrm{MHz}$ and modify it with a tall resonance suppression of $85 \%$ at $33.33 \mathrm{MHz}$ in shape of an inverse Gauss function and a simple rectangular cut-out inside the same frequency range -31 to $36 \mathrm{MHz}$. Figure 9 shows the results in spatial and time domain with a time base of $200 \mathrm{~ns}$. The influence of the resonance suppression to the cosine-pulse in time domain is clearly visible, but a difference between the two ways of suppression is missing in this time base. Careful checking unwraps, however, there is a difference (not displayed in the figure), but it is very small and appears after about $8 \mu \mathrm{s}$, far outside our time span of interest. We can summarize, the implementation of a transducer resonance suppression is possible and useful with any excitation function for optimum use of the receiver sensitivity. To use the
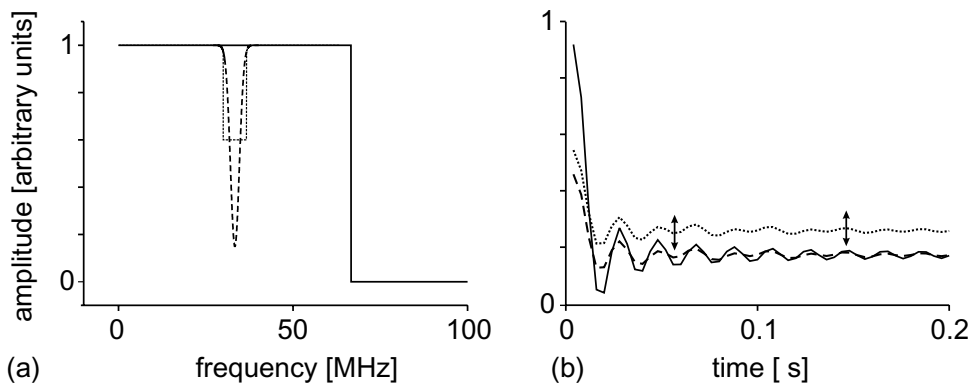

Figure 9. Cosine-pulses calculated by FFT from the low-pass characteristics in spatial domain. The transducer resonance is suppressed by an inverse Gauss-curve shaped or a rectangular $85 \%$ amplitude $5 \mathrm{MHz}$ broad cut-out of around $33.33 \mathrm{MHz}$. The resonance suppression has a strong impact on the pulse in time domain. The effect of the cut-out shape (inverse Gauss-curve or rectangular) is very small. Both pulses only differ slightly after $8 \mu \mathrm{s}$, not displayed in the figure. Only for demonstration and to be able to compare the curves for both ways of resonance suppression the "rectangle" is displayed with offset. —, no resonance suppression; ---, Gauss resonance suppression; ...., rectangle resonance suppression. 
exact resonance curve of system might be less important, because the difference between the different shaped cut-outs is very minor. Therefore, our first simple approach, forming the excitation function from the available monochromatic waves, already worked very successfully with results comparable to those of the more sophisticated functions.

\subsubsection{Evaluation of the data transfer function}

Contrary to the sweep technique (600 files of $4 \mu$ s duration and 2048 data points) the received DTF should be saved with a much longer time base and a much higher resolution. This is plausible, because the amount of information spread out over several hundreds of files for the sweep technique is now concentrated in one single file. We saved about $40 \mu \mathrm{s}$ with a resolution of 65,536 data points. Li et al. (2002) published $50 \mu$ s and 100,000 data points. With increased resolution the results of the evaluation of the DTF improves, i.e. the reproduction of the monochromatic signals. The received DTF is the response of the system to the excitation function containing all monochromatic frequencies between the upper and lower cut-off frequencies.

Convolving vectors $u$ and $v$ means, algebraically, the same operation as multiplying the polynomials whose coefficients are the elements of $u$ and $v$. If $m=\operatorname{length}(u)$ and $n=$ length $(v)$, then $w$ is a vector of length $m+n-1$ whose $k$ th element is

$$
w(k)=\sum_{j} u(j) v(k+1-j)
$$

The sum is over all the values of $j$ which lead to legal subscripts for $u(j)$ and $v(k+1-j)$, specifically $j=\max (1, k+1-n): \min (k, m)$. The convolution theorem says, roughly that convolving two sequences is the same as multiplying their Fourier transforms. In order to make this precise, it is necessary to pad the two vectors with zeros and ignore round-off error.

$$
f \cdot g \leftrightarrow F \otimes G
$$

That means, reproduction of all the analogues of the monochromatic signals received and saved by the sweep technique require the stepwise convolution of the DTF with each of the monochromatic frequencies. Corresponding to Eq. (12) the time axis has to be re-scaled by the factor $C$.

$$
C=\frac{\left(m+n_{i}-1\right)}{m}
$$

with $m$ the length of the DTF and $n_{i}$ the length of the monochromatic signal amplitude.

The signal has to be strictly monochromatic. Otherwise the convolution will fail in reproducing the response of the system for this single frequency, because a nonmonochromatic oscillation consists of more than one frequency peaks in spatial domain, i.e. multiplying in spatial domain would be no longer effective for selection. That also means the sampling frequency for the DTF and the signal must satisfy the Nyquist/Shannon sampling theorem (see Eq. (5)): "A signal can be properly reconstructed from its samples if the original signal is sampled at a frequency that is greater than twice 


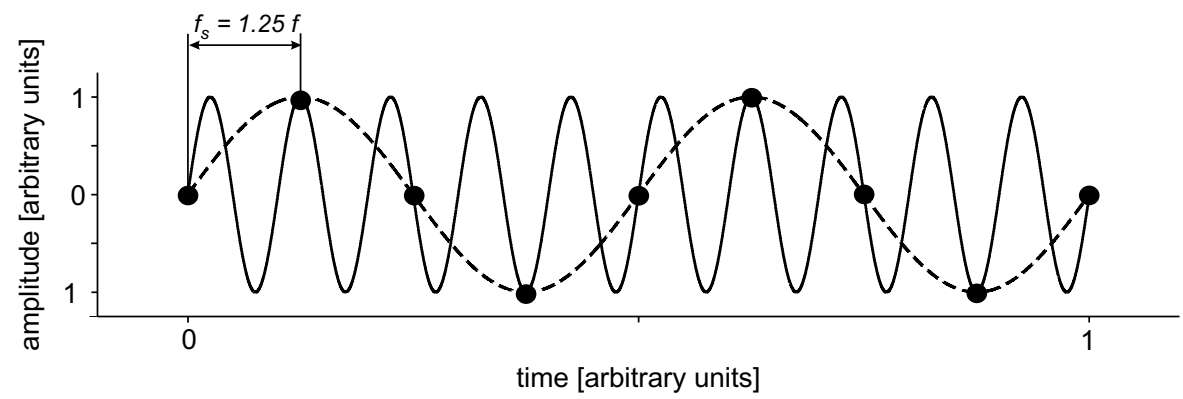

Figure 10. Violation of the Nyquist theorem, i.e. the sampling frequency has to be twice the signal frequency at the minimum, results in failing the reproduction of the original analog signal from the digitized signal. The displayed lower frequency also matches all data points. During the reproduction from the digital file this lower frequency will also appear. In computer graphics the effect results in the wellknown Moiré patterns and aliasing (modified from Seidel and Myszkowski, 2004).

the highest frequency component in its spectrum."

$$
f<f_{\mathrm{Ny}} \equiv \frac{1}{2} f_{\mathrm{s}}
$$

with $f$ the signal frequency, $f_{\mathrm{Ny}}$ the Nyquist frequency, and $f_{\mathrm{s}}$ the sampling frequency.

Otherwise the signal reproduced from the file do not represent the original signal. The data also represent another signal of lower frequency (see Fig. 10). We all know the effect from digital cameras. In digital photography and computer graphics the effect is called aliasing and results in Moiré patterns, non-existing in the original optical images.

Figure 11 compares a $36 \mathrm{MHz}$ signal saved by sweep technique with the corresponding signal reproduced from the transfer function by convolution of the same experiment. The time base was adapted corresponding Eq. (14). The signals match to a great extent. Analog to the sweep technique the reproduced signals are further processed as published by Q2 Knoche et al. (1997, 1998), Li et al. (1998), Shen et al. (1998), Mueller et al. (2002, 2004). The DTF technique reduces the time for one velocity measurement from more than $30 \mathrm{~min}$ to a few seconds, but shifts the efforts from the measurement itself to the subsequent evaluation. Transient measurements without significant reduction of the precision are possible, if the response is measured with high resolution, because the response to all frequencies is recorded at the same time.

\subsection{Sample preparation}

\subsubsection{San Carlos olivine}

San Carlos olivine is widely used for equation-of-state investigations to derive reliable compositional constraints from observed seismic velocity profiles, because it is a good representation of the most abundant mineral in the upper mantle (Chen et al., 1996; Chang-Sheng-Zha et al., 1998). Consequently it was ideal for testing a new method, as the results can be compared with published data. The samples came from San Carlos, an ultramafic inclusion locality, about $30 \mathrm{~km}$ east of Globe, Arizona. A detailed petrological 


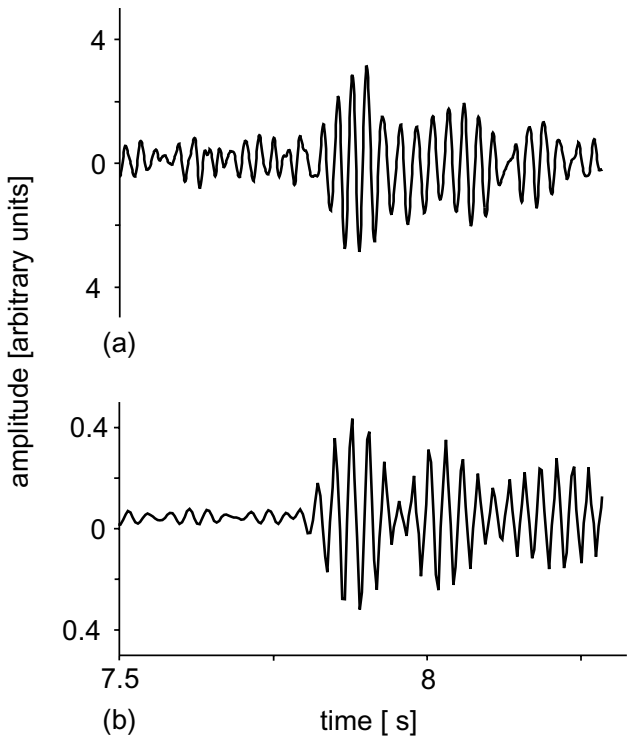

Figure 11. Comparison of a $39 \mathrm{MHz}$ signal, saved during a sweep technique (b) measurement with the corresponding signal, reproduced by convolving (a) the data transfer function with a monochromatic $39 \mathrm{MHz}$ oscillation.

and geochemical description is given by Frey and Prinz (1978). In the terminology of Frey and Prinz, two different types of xenoliths occur. All xenoliths investigated belong to group I. A recrystallized or annealed near-equilibrium texture is characteristic for all samples. The grain size of recrystallized olivine grains is about $30-35 \mu \mathrm{m}$. A few grains with wavy extinction are about several hundred micrometers in diameter (Wirth, 1996).

\subsubsection{Anorthite}

The anorthite samples were manufactured by E. Rybacki from crushed $\mathrm{CaAl}_{2} \mathrm{Si}_{2} \mathrm{O}_{8}$ glass powder, which was hot-isostatically pressed and crystallized at $300 \mathrm{MPa}$ confining pressure and temperatures between 900 and $1200^{\circ} \mathrm{C}$ in a Paterson gas pressure apparatus. The original sample size was $10 \mathrm{~mm}$ diameter and $20 \mathrm{~mm}$ length. The porosity was less than $1 \mathrm{vol} . \%$, the density $2.75 \mathrm{~g} / \mathrm{cm}^{3}$. The grains are prismatic with an average aspect ratio of about 2.7. Twins are abundant. The (arithmetic) mean grain size is $3.4 \pm 0.2 \mu \mathrm{m}$ (for details see Rybacki and Dresen, 2000).

The grain size of the samples was measured by scanning electron microscopy. The samples of the three materials were shaped with a high-precision $( \pm 0.5 \mu \mathrm{m})$ cylindrical grinding machine.

\subsubsection{Clinoenstatite}

For the following high-pressure experiments LCEn powder was synthesized from a gel with a molar ratio of $\mathrm{MgO}: \mathrm{SiO}_{2}=1: 1$ by heating up to $1500^{\circ} \mathrm{C}$ for $2 \mathrm{~h}$ followed by 
quenching of the material to room temperature at $1 \mathrm{bar}$. The run product was single-phase LCEn as characterized by XRD. The cell parameters at normal conditions were determined using Rietveld refinement with the program package GSAS (General Structure Analysis System) and are as follows: $a=9.6033(1) \AA, \quad b=8.8142(1) \AA$, $c=5.16933(7) \AA, \beta=108.304(1), V=415.42(1) \AA^{3}$, the $1 \sigma$ uncertainty of the last digit derived from Rietveld refinement is given in parentheses. The cell dimensions of LCEn are in excellent agreement with data published by Ohashi (1984) and Angel and Hugh-Jones (1994).

LCEn-powder was pressed to cylindrical samples of $2 \mathrm{~mm}$ in length and diameter. Together with the sodium chloride calibrant manufactured from powder of $99.5 \%$ purity (analytical grade by Merck) and a medium grain size of $50 \mu \mathrm{m}$ using the same powder press, the sample was contained in a hBN ring to effectively reduce non-hydrostatic stress. To test the influence of local stress concentrations of the observations, a portion of the LCEn powder and the highly ductile hexagonal BN-powder were mixed in a volume ratio of 1:1 and a sample rod was pressed from this material (run 3/26).

Ultrasonic measurements in the $50 \mathrm{MHz}$ frequency and $50 \mu \mathrm{m}$ grain-size range require sample porosity less than $1 \%$, because gas-filled pores act as scattering centers for the ultrasonic waves. The only way to get such very low-porosity samples was hot-isostatic pressing, by using MAX80. HIP requires a pressure of at least $0.2 \mathrm{GPa}$, and an optimum temperature treatment to close the initial pore space of about $20 \mathrm{vol} . \%$, still existing after cold pressing, by recrystallization without grain growth. A modified $8 \mathrm{~mm}$ standard set-up for non-ultrasonic experiments (Fig. 12) was used for the HIP-treatment of pure LCEn powder at $0.4 \mathrm{GPa}$ and $1400^{\circ} \mathrm{C}$ for $2 \mathrm{~h}$. At temperatures above $1557^{\circ} \mathrm{C}$ LCEn melts incongruently (Bowen and Andersen, 1914). The first runs produced samples that segmented to disks of about $0.5 \mathrm{~mm}$ length. Following the advice of B. Li (personal communication, 2002) further quenching was conducted at $40 \mathrm{~K} / \mathrm{min}$ which inhibited segmentation. The HIP samples were shaped to cylinders of $2 \mathrm{~mm}$ diameter and $1.2 \mathrm{~mm}$ length with a high-precision cylindrical grinding machine and polished at both the end faces. Parts of the HIP sample were examined by XRD to rule out any phase transition.

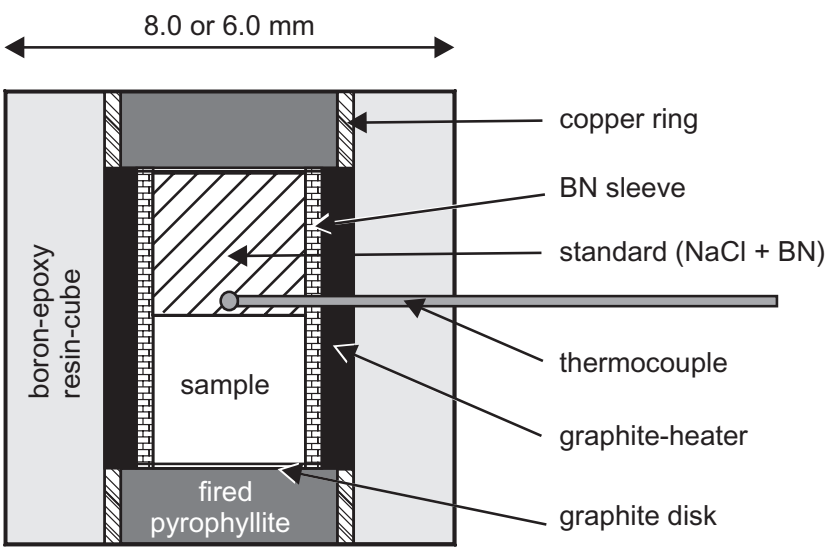

Figure 12. Modified standard cell assembly for $6 \mathrm{~mm}$ anvil, truncation HIP-ping of low-porosity samples at MAX80. 


\section{Results and analysis}

\subsection{San Carlos olivine}

Figure 13 shows the results for ultrasonic compressional and shear waves of San Carlos olivine up to $3 \mathrm{GPa}$ pressure using a symmetrical as well as an asymmetrical configuration.

Both elastic wave velocities were corrected for the elastic sample shortening by calculating the compressibility by successive approximation from our $v_{\mathrm{p}}$ and $v_{\mathrm{s}}$ data Q6 (Mueller et al., 2000). This approximation converges according to Banach's fixpoint theorem for meaningful $v_{\mathrm{p}}$ and $v_{\mathrm{s}}$ data. Using this method we derived velocity data independent of literature data and any sample comparison. For details of sample length Q1 measurements in multi-anvil devices, see, e.g. Mueller et al., pp. xxx, this volume. This procedure was checked by X-radiography.

In addition to our results for both cell assemblies, Figure 13 also shows the values of $c_{22}$, $c_{33}, c_{44}, c_{55}$ and $c_{66}$, published by Webb (1989), Zaug et al. (1993) and Chen et al. (1996).
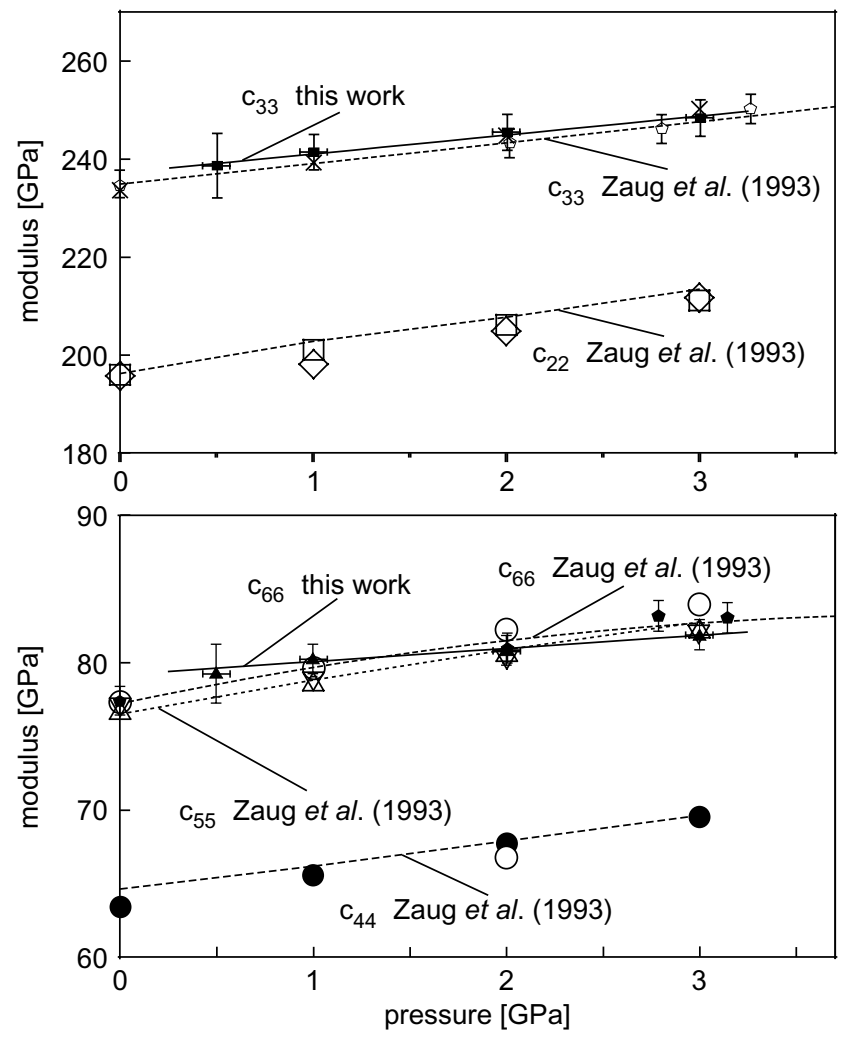

Figure 13. Elastic moduli of San Carlos olivine versus pressure up to $3 \mathrm{GPa}$ measured in the multi-anvil apparatus MAX80. Published data (Webb, 1989; Zaug et al., 1993; Chen et al., 1996) are plotted for comparison. $\square, c_{33}$ this study; $\boldsymbol{\Lambda}, c_{66}$ this study; $\square, c_{33}$ Zaug et al. (1993);,$c_{66}$ Zaug et al. $(1993) ; \times, c_{33}$ Webb (1989); $\square, c_{22}$ Webb (1989);,$c_{44}$ Webb (1989) (mode 4); $\triangle c_{55}$ Webb (1989) (mode 5); $\bigcirc, c_{66}$ Webb (1989) (mode 12); $\diamond, c_{22}$ Chen et al. (1996); $\nabla, c_{55}$ Chen et al. (1996). 
The solid line is the linear least square best fit for our data. The dashed line is the secondorder polynomial least square best fit for the data of Zaug et al. (1993). Our data for the [001] direction are in agreement with the published data within the limit of experimental errors $(\sim 1.5 \%)$. The measurements with the asymmetrical configuration were performed at a sample manufactured in a direction of $36^{\circ}$ from the [001] direction at the [100] plane. Consequently the modulus derived from the compressional wave velocity (not displayed) has a value between the published data for $c_{22}$ and $c_{33}$, whereas published $c_{55}, c_{66}$ and the module, calculated from our corresponding transverse wave data correspond to each other in the limits of experimental uncertainty $(\sim 1.5 \%)$. The data were compared by an in-house program (Schilling, 1998) using a solution for the Christoffel equation. The reference data were taken from Landolt-Bömstein (Hearmon, 1984).

\subsection{Anorthite}

Figure 14 compares our high pressure $v_{\mathrm{p}}$ and $v_{\mathrm{s}}$ data (solid line) for polycrystalline anorthite with hydrostatic high-pressure results, measured up to $0.75 \mathrm{GPa}$ at polycrystalline samples, HIP-ped at $1.5 \mathrm{GPa}$ and $1000^{\circ} \mathrm{C}$ in a piston-cylinder apparatus, published by

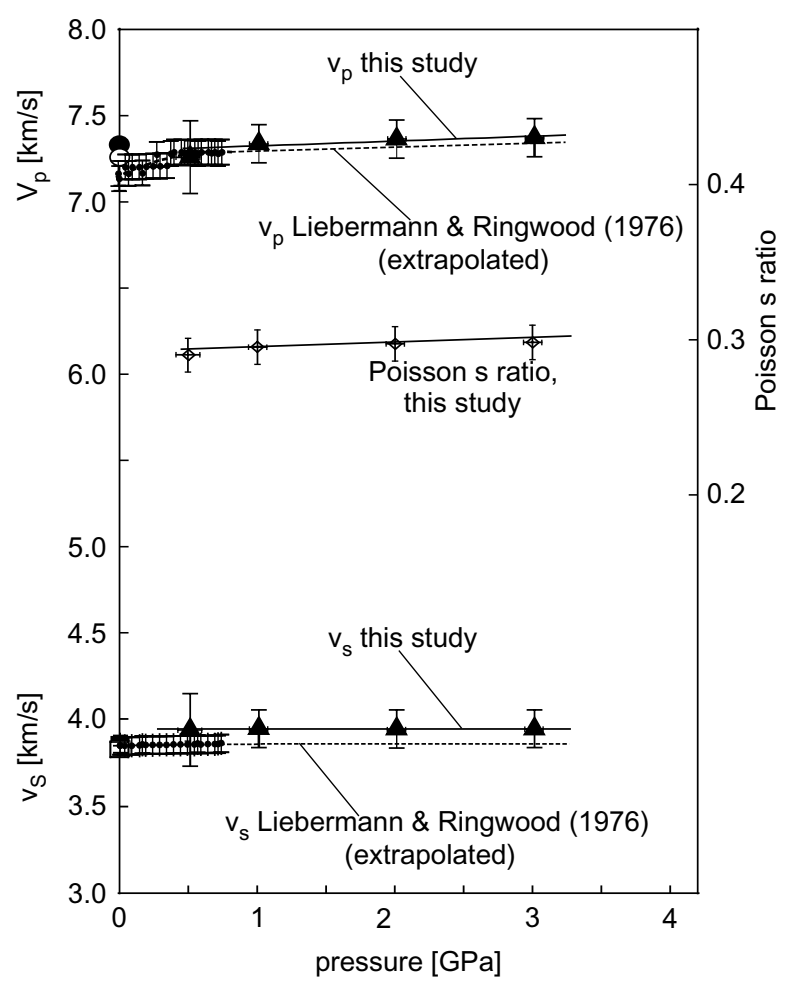

Figure 14. $v_{\mathrm{p}}, v_{\mathrm{s}}$ and Poisson's ratio $\nu$ of anorthite versus pressure up to $3 \mathrm{GPa}$ measured in the multianvil apparatus MAX80. Published data (Liebermann and Ringwood, 1976; Bass, 1995) are plotted for comparison. - arithmetic mean value of $v_{\mathrm{p}}$ (Bass, 1995); $\bigcirc$, geometric mean value of $v_{\mathrm{p}}$ (Bass, 1995); $\square$, arithmetic mean value of $v_{\mathrm{s}}$ (Bass, 1995); $\square$, geometric mean value of $v_{\mathrm{s}}$ (Bass, 1995). 
Liebermann and Ringwood (1976, dashed line) and with the arithmetic and geometric mean values of $v_{\mathrm{p}}$ and $v_{\mathrm{s}}$ deduced from normal-pressure elastic moduli published by Gebrande (1982) and Bass (1995). Our graphs correspond to the normal-pressure data (Bass, 1995) and to the high-pressure data up to $0.75 \mathrm{GPa}$ of Liebermann and Ringwood (1976) within the limits of experimental errors $(\sim 1.3$ to $1.7 \%)$. Our pressure derivatives (linear best fit) also correspond to the extrapolated high-pressure data of Liebermann and Ringwood (1976) (second-order polynomial best fit) within these limits. The unusual pressure independency of the shear wave velocity, i.e. $v_{\mathrm{s}}$ is constant with pressure within the precision of the pulse transmission technique $( \pm 0.05 \mathrm{~km} / \mathrm{s})$, described by Liebermann and Ringwood (1976) is validated by our measurements for polycrystalline samples. The slight deviation to the absolute values might be caused by the fact that the remaining pore space in our sample might be smaller and that a more uniform crystallization seems to be achieved, due to hot-isostatic pressing in a Paterson apparatus. The central graph is the Poisson's ratio, about 0.29 at room conditions with a slight increase to 0.30 at $3 \mathrm{GPa}$ pressure at $20^{\circ} \mathrm{C}$, determined from the presented velocity data. The sample shortening under pressure was derived from the compressibility the same way as described in Section 3.1.

\subsection{Clinoenstatite}

Regression analysis (Belsley et al., 1980; Holland and Redfern, 1997) was used for latticerefining the energy-dispersive X-ray data of HCEn and LCEn determined with MAX80 at HASYLAB (see Fig. 15). The method is capable of determining lattice parameters with high resolution from the in situ X-ray results, i.e. powder-diffraction data from a beam of white synchrotron radiation impinging on a small sample surrounded by heater, electrical insulator and gasket material. The narrow slits between the X-ray absorbing anvils result in an observation of a limited part of the diffraction cone. The regression diagnostics refinement is based on minimization of the differences between the measured $d_{h k l}$ and its calculated values. The modeling was performed using the program UnitCell from Department of Earth Sciences, Cambridge University.

We started the high pressure/high temperature experiments (run 3/24, see Fig. 16) using the set-up shown in Figure 12 with a sample of pure LCEn powder. By raising the pressure above $6.5 \mathrm{GPa}$ at RT pure HCEn was formed. The phase transition was observed by in situ XRD measurements. The pressure and temperature of the first appearance of LCEn in the $\mathrm{X}$-ray diffraction pattern was determined by successively raising the temperature in steps of $50 \mathrm{~K}$ at a given $P$ (Fig. 16). This procedure was performed for three different $P$ conditions, 6.61(5), 7.20(5) and 7.50(5) GPa. We used the reaction from HCEn-LCEn to determine the phase boundary, as this reaction is kinetically less hindered than the back-reaction.

Run 3/25 (see Fig. 16) is the continuation of run 3/24 at a pressure of 7.89(5) GPa. To ensure that the results are not distorted by hysteresis effects because of multiple crossing the phase boundary back and forth, we only crossed the phase boundary once by increasing temperature in this experiment. Figure 15 shows the energy-dispersive XRD spectra of HCEn and LCEn as measured in MAX80 under in situ conditions at 6.61(5) GPa and 250 and $300^{\circ} \mathrm{C}$, respectively. During the phase transition the position of the strongest 

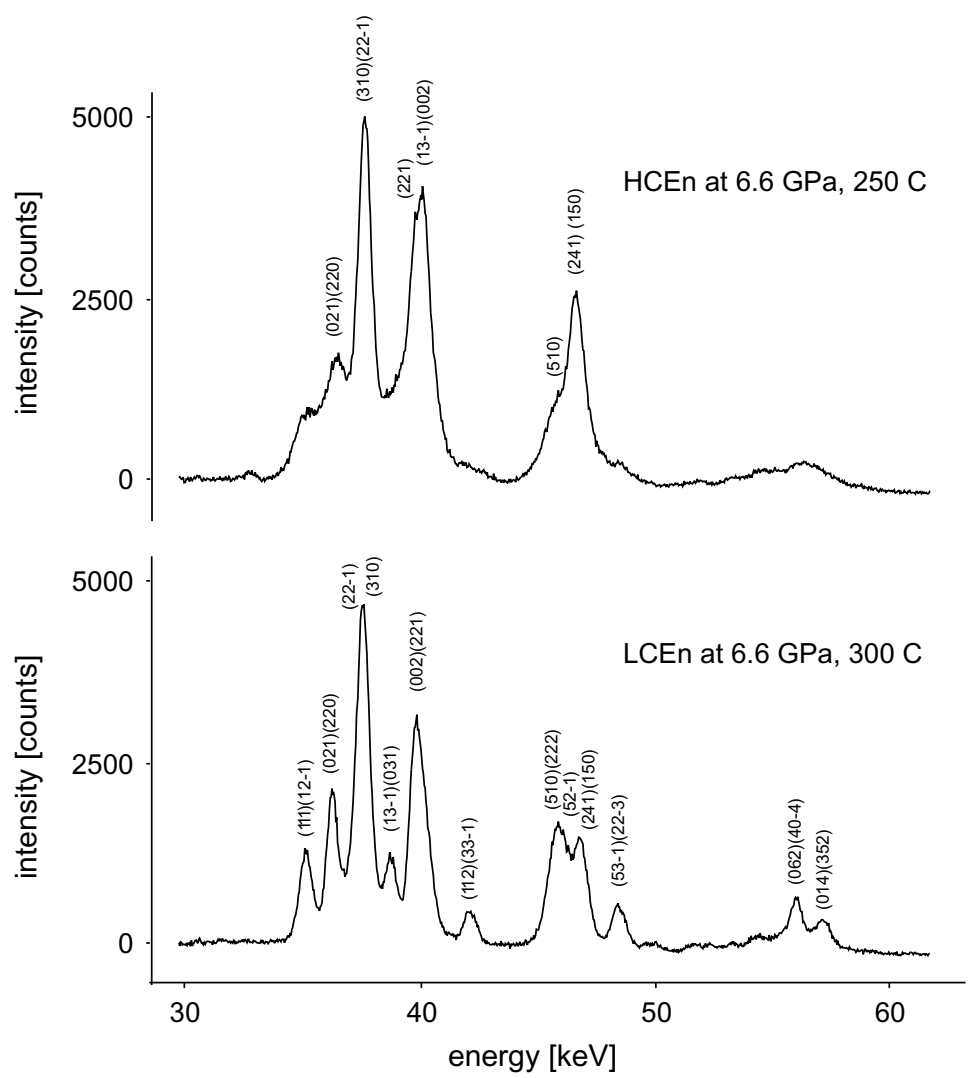

Figure 15. XRD data for HCEn and LCEn at 6.6 GPa and $250 / 300^{\circ} \mathrm{C}$, measured in MAX80. The stronger peaks have a limited significance for phase detection because the energy shift is very small. Several smaller peaks are used for phase identification.

diffraction lines change by only a small amount. However, between four and seven diffraction lines with lower intensity could be used to securely distinguish between LCEn and HCEn.

Run 3/26 (see Fig. 16) reproduced the $P, T$ regime of run $3 / 24$ with a slightly higher pressure, but the sample was a 1:1 per volume mixture of HCEn and hBN. The phase boundary was crossed at pressures of 6.74(5), 6.93(5) and 7.28(5) GPa, respectively. Due to the dilution of $\mathrm{CEn}$ in $\mathrm{BN}$, the detected intensity of the diffraction lines was less for the two-component samples in run $3 / 26$, but still sufficient for the evaluation. The experiment with the mixed sample gave slightly higher pressures for the phase boundary. This might be a result of the different compressibility of both constituents of the mixture, resulting in lower pressure in the more compressible medium, as discussed by Dietrich and Arndt (1982) and Will et al. (1982). Consequently, the data of the first cycle of run 3/24 and run $3 / 25$ mostly define the maximum temperature conditions of the phase boundary as shown in Figure 16. The solid line between the last existence of HCEn and the first appearance of LCEn is the best fit to our results. Our results only represent the minimum $P$ conditions of the HCEn-LCEn phase boundary, which is approximated by $P(\mathrm{GPa})=0.0021\left(\mathrm{GPa} /{ }^{\circ} \mathrm{C}\right)$ 


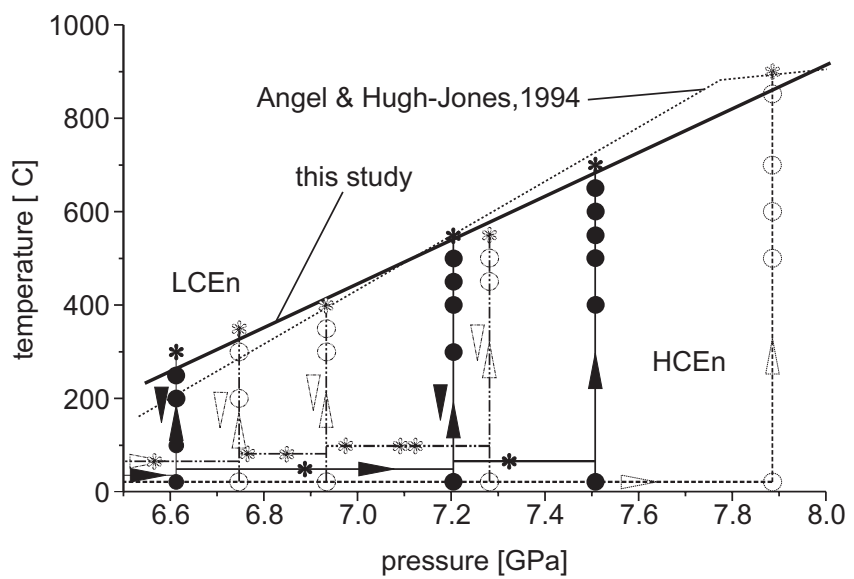

Figure 16. Scheme of experimental runs to determine the HCEn-LCEn phase boundary. Run 3/24 and $3 / 26$ crossed the phase boundary HCEn-LCEn three times. The arrows indicate the pressure temperature path of the experiments. By raising the pressure above 6.5 GPa at RT HCEn was formed followed by increasing the temperature in steps of $50 \mathrm{~K}$ up to the first existence of HCEn. Then the sample was cooled by switching off the power and the pressure was increased to form HCEn again. To rule out hysteresis effects run $3 / 25$ crossed the phase boundary only once. Run 3/26 used a sample mixture of clinoenstatite and hBN 1:1. The solid line represents our results of the maximum temperature condition of the HCEnQ8 LCEn phase boundary. The dotted line represents the data published by Angel and Hugh-Jones (1997). - , run $3 / 24(100 \%$ CEn); $\cdots$, Run 3/25 (100\% CEn); ---, run 3/26 (50\% CEn + 50\% hBN); -, this study; $*$, LCEn; •, HCEn.

$T\left({ }^{\circ} \mathrm{C}\right)+6.048(\mathrm{GPa})$. Nevertheless, our results (see Fig. 16) fall within the pressure range determined by Angel and Hugh-Jones (1994) at ambient conditions. The invariant point defined by the intersection of the HCEn-LCEn equilibrium determined within this study is in good accordance with the invariant point deduced by OEn-LCEn reaction after Angel and Hugh-Jones (1994) which lies at about $7.9 \mathrm{GPa}$ and $865^{\circ} \mathrm{C}$. This is contrast to the experimental results of Kanzaki (1991) and Ulmer and Stalder (2001).

Figure 17 compares our cell parameters with the results of Angel and Hugh-Jones (1994) and Shinmei et al. (1999) for HCEn at ambient temperature and with the results of Shinmei et al. (1999) for HCEn at maximum temperature near the phase transition, see also Table 1. The results are in good agreement in the limits of the $2 \sigma$ experimental uncertainty. The unit-cell volumes (Table 1) of this study correspond to those determined by Shinmei et al. (1999) within a multi-anvil press and at pressures $<7 \mathrm{GPa}$ with those by Angel and Hugh-Jones (1994) from as diamond anvil study using synthetic single crystals of CEn.

Figure 18 shows the results of our ultrasonic experiments with a HIP-ped clinoenstatite sample. Similar to run $3 / 24$ (see Fig. 16) we targeted to transform the sample to a minimum porosity HCEn sample by raising the pressure at normal temperature up to 6.7 GPa. Because there is some indication that the sample was not completely transformed to HCEn before passing the phase boundary to LCEn between 250 and $300^{\circ} \mathrm{C}$ we do not report this ultrasonic results. A further temperature increase up to $700^{\circ} \mathrm{C}$ ensured representative ultrasonic data, because the measurements started deep inside the 

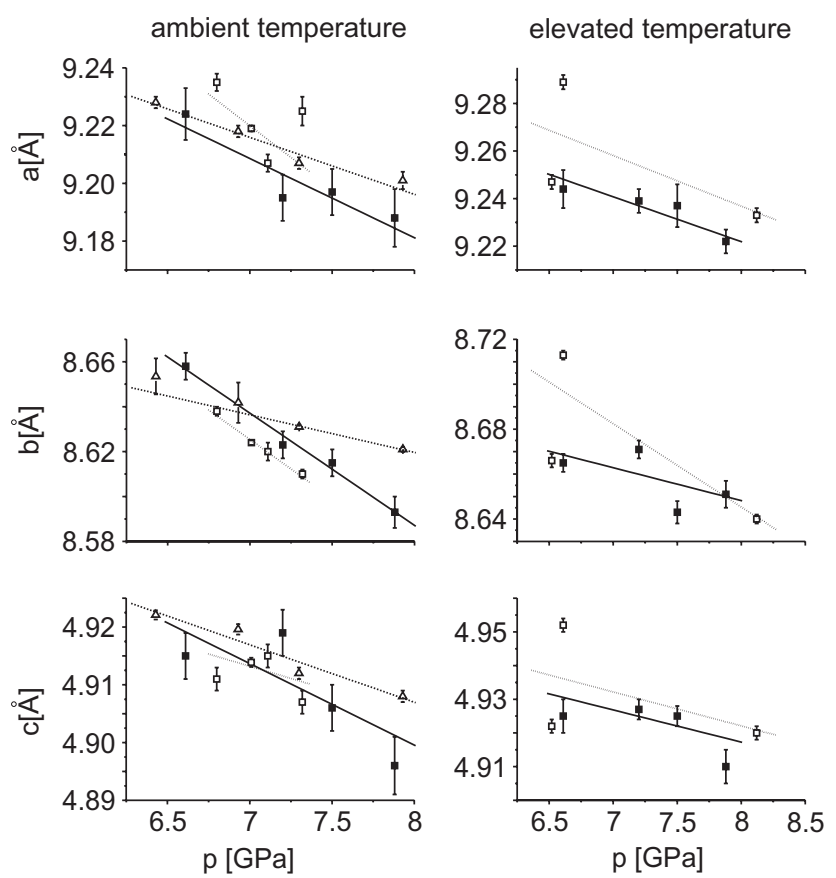

Figure 17. Variation of HCEn unit cell parameters with pressure at RT and at elevated temperatures, close to the HCEn-LCEn phase boundary. The results of this study (ם) (-) (see also Table 1) are compared with the data published by Shinmei et al (1999)( $\square)(\ldots)$ and Angel and Hugh-Jones (1994) $(\triangle)$ (- - ). The lines represent the least square linear best fit for the data sets of this study and of the comparative authors.

LCEn-stability field and the sample never left it during the following pressure increase up to $7.5 \mathrm{GPa}$. The gradual temperature increase at constant pressure load also targets a minimum deviatoric stress inside the sample. The displayed best fit lines represent the velocity dependence on pressure at constant temperature of $700^{\circ} \mathrm{C}$ for LCEn. For $v_{\mathrm{p}}$ and $v_{\mathrm{s}}$ a temperature derivative at $700^{\circ} \mathrm{C}$ of 0.8 and $0.7 \mathrm{~km} /(\mathrm{s} \mathrm{GPa})$ was determined, respectively. The ultrasonic measurements were performed using the new developed ultrasonic transfer function DTF technique. To compare the results performed at 6.7 and $7.5 \mathrm{GPa}, v_{\mathrm{p}}$ and $v_{\mathrm{s}}$ were also measured using the classical sweep technique. The data are in good agreement.

Recently Kung et al. (2004) published the results of very thorough and innovative experiments on elastic wave velocities at the orthopyroxene-HCEn transformation, as a systematic continuation of the measurements of Flesch et al. (1998) with orthopyroxene up $10 \mathrm{GPa}$. Different from our experiments an OEn sample entered the HCEn-stability field at much higher pressures and temperatures of about $16 \mathrm{GPa} / 650^{\circ} \mathrm{C}$.

The LCEn-HCEn phase transition might be an important reaction in deeper parts of cold, fast subducting slabs, where the temperature increase is retarded. Our preliminary results indicate a velocity drop of less than $0.5 \%$ within a cold, fast subducting pyrolitic mantle.

During the transformation of the 1:1 sample a strong reduction in porosity is observed. This indicates that at the phase transition the rheological behavior of the sample allows a 
1013

1014

1015

1016

1017

1018

1019

1020

1021

1022

1023

1024

1025

1026

1027

1028

1029

1030

1031

1032

1033

1034

1035

1036

1037

1038

1039

1040

1041

1042

1043

1044

1045

1046

1047

1048

1049

1050

1051

1052

1053

1054

1055

1056

1057

1058

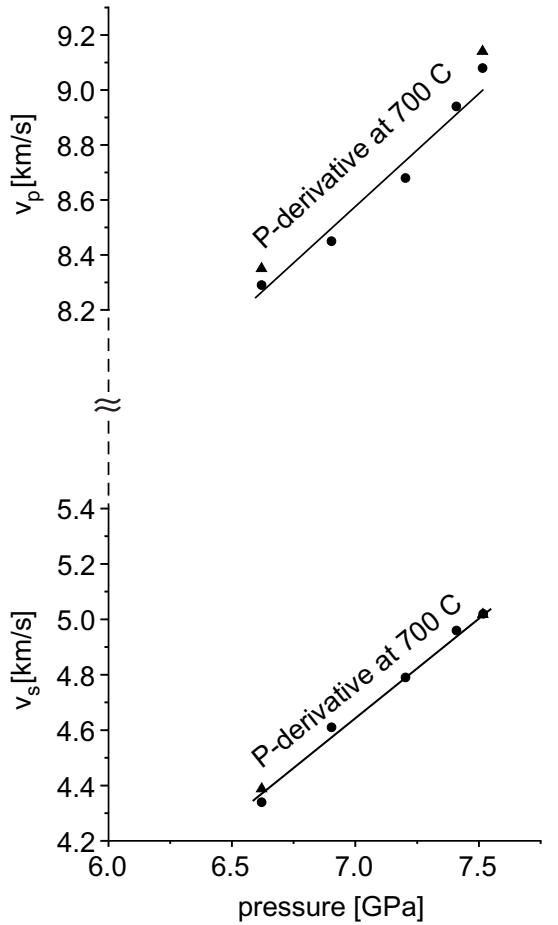

Figure 18. Compressional and shear wave velocities, $v_{\mathrm{p}}$ and $v_{\mathrm{s}}$, in LCEn in dependence on pressure at $700^{\circ} \mathrm{C}$. The displayed results of run $3 / 52$ were measured using both interferometric techniques (see text for details). The data $(\bullet)$ represent the elastic wave velocity values at $700^{\circ} \mathrm{C}$, measured by the DTF technique in dependence on pressure between 6.6 and 7.5 GPa. To compare the results of the DTF method with the classical sweep technique $(\Delta), v_{\mathrm{p}}$ and $v_{\mathrm{s}}$ were also measured by both methods at 6.6 and $7.5 \mathrm{GPa}$.

modification of its microstructure. This behavior linked to the CEn phase transformation can be explained by transformation plasticity (e.g. Poirier, 1982; Schmidt et al., 2002). Therefore, a reduced shear strength related to the CEn transition might result in a markedly reduced viscosity of CEn-bearing rocks and should influence the rheology of the lithospheric mantle of down-going slabs.

\section{Conclusions}

The results show the power of the demonstrated ultrasonic interferometric measurements in conjunction with XRD in multi-anvil devices under simulated Earth's mantle conditions. The results for San Carlos olivine and HIP-polycrystalline anorthite were compared with published data and illustrate the accuracy and reliability of the method. The results for clinoenstatite demonstrate the potential of simultaneous elastic and X-ray measurements to study unquenchable phase transitions.

For the optimum adaptation to different samples and experimental conditions several cell assemblies and the corresponding anvils were developed and tested under 
high-pressure conditions. The modification of MAX80 for ultrasonic measurements had no negative side effect on the experimental limits of the high-pressure/high-temperature apparatus. In addition to the interferometric sweep method a DTF technique was developed and optimized for MAX80. The coincidence of the results from both techniques could be demonstrated by a combined experiment (3/52), i.e. both techniques were used for the same sample, during the same experiment, under the same pressure/temperature conditions. Together with the newly developed X-radiography for in situ deformation Q1 measurements (see Mueller et al., pp. xxx, this volume) the DTF technique allows extensive transient measurements, because this way ultrasonic interferometry changed from the most limiting technique for the experiment to the fastest one of the applied methods. This has a fundamental meaning for future experiments, because the kinetics of phase transitions is accessible for elastic wave velocity measurements now. Experiments with complex phase assemblages, unquenchable phases, volatile-saturated and molten systems will dramatically improve the scientific output of high-pressure research for the interpretation of geophysical data and the dynamical understanding of the interior of Earth and other planetary bodies.

\section{Acknowledgements}

We would like to express our special thanks to the editors J. Chen, Y. Wang, T. Duffy, G. Shen, and L. Dobrzhinetskaya for their initiative and patience, as well as two unknown references for their very helpful reviews. Especially without the very constructive guidance of Y. Wang the chapter would not exist in the presented form. Our special thank is for B. Li. Without his helpful and fruitful discussions the demonstrated development would have been much less successful. We also acknowledge the kind guidance and the support of B. Liebermann, D. Weidner and M. Vaughan. The authors are particularly indebted to B. Wunder, R. Milke and E. Rybacki for sample courtesy, including the sample HIP by the latter, as well as for their technical support; M. Kreplin, G. Berger and S. Gehrmann for the special preparation as well as all colleagues of both mechanical workshop for their dedicated support.

\section{References}

Angel, R.J., Hugh-Jones, D.A., 1994. Equations of state and thermodynamic properties of enstatite pyroxenes. J. Geophys. Res. 99 (B10), 19777-19783.

Angel, R.J., Ross, N.L., 1996. Compression mechanisms and equation of state. Philos. Trans. R. Soc. London, A 354, 1449-1459.

Angle, R.J., Chopelas, A., Ross, N.L., 1992. Stability of high-density clinoenstatite at upper-mantle pressures. Nature 358, 322-324.

Bass, J.D., 1995. Elasticity of minerals, glasses and melts. In: Ahrens, Th.J. (Ed.), Mineral Physics and Crystallography: A Handbook of Physical Constants, AGU Reference Shelf 2, pp. 45-63.

Beaulieu, N.C., Tan, C.C., Damen, M.O., 2001. A "better than" Nyquist pulse. IEEE Commun. Lett. 5 (No. 9), 367-368.

Belsley, D.A., Kuh, E., Welsh, R.E., 1980. Regression Diagnostics: Identifying Influential Data and Sources of Colinearity. Wiley, New York.

Birch, F., 1960. The velocity of compressional waves in rocks to 10 kilobars. Part 1. J. Geophys. Res. 65, $1083-1102$. 
Birch, F., 1961. The velocity of compressional waves in rocks to 10 kilobars. Part 2. J. Geophys. Res. 66, 2199-2224.

Bowen, N.L., Andersen, O., 1914. The binary system MgO-SiO 2 . Am. J. Sci. 37, 487-500.

Chan, H.A., 2004. EEE482F Telecommunication. Pulse Shaping. [online] [cited 2004-04-02]. Available from: http://www.eng.uct.edu.za/ achan/eee482f/note/eee482f-0009.ppt.

Chang-Sheng-Zha, Duffy, T.S., Downs, R.T., Ho-Kwang-Mao, Hemley, R.J., 1998. Brillouin scattering and X-ray diffraction of San Carlos olivine: direct pressure determination to $32 \mathrm{GPa}$. Earth Planet. Sci. Lett. 159 (1-2), 25-33.

Chen, G., Li, B., Liebermann, R.C., 1996. Selected elastic moduli of single-crystal olivines from ultrasonic experiments to mantle pressures. Science 272, 979-980.

Decker, D.L., 1971. High-pressure equation of state for $\mathrm{NaCl}, \mathrm{KCl}$, and CsCl. J. Appl. Phys. 42, 3239-3244.

Dietrich, P., Arndt, J., 1982. Effect of pressure and temperature on the physical behavior of mantlerelevant olivine, orthopyroxene and garnet. In: Schreyer, W. (Ed.), High-Pressure Researches in Geoscience. E. Schweizerbart'sche Verlagsbuchhandlung, Stuttgart, pp. 293-306.

Ekbal, A., 2004. Receiver SNR and Nyquist pulses, [online]. [Cited 2004-02-03]. Portable Document Format. Available from: http://www.stanford/edu/class/ee379a/handouts/lec8.pdf.

Flesch, L.M., Li, B., Liebermann, R.C., 1998. Sound velocities of polycrystalline $\mathrm{MgSiO}_{3}$-orthopyroxene to $10 \mathrm{GPa}$ at room temperature. Am. Mineral. 83, 444-450.

Frey, F.A., Prinz, M., 1978. Ultramafic inclusions from San Carlos, Arizona: petrologic and geochemical data bearing on their petrogenesis. Earth Planet. Sci. Lett. 38, 129-176.

Funamori, N., Yagi, T., Uchida, T., 1996a. High-pressure and high-temperature in situ x-ray diffraction study of iron to above $30 \mathrm{GPa}$ using MA8-type apparatus. Geophys. Res. Lett. 23 (No. 9), 953-956.

Funamori, N., Yagi, T., Utsumi, W., Kondo, T., Uchida, T., 1996b. Thermoelastic properties of $\mathrm{MgSiO}_{3}$ perovskite determined by in situ x-ray observations up to $30 \mathrm{GPa}$ and $2000 \mathrm{~K}$. J. Geophys. Res. 101, $8257-8269$.

Gebrande, H., 1982. Elasticity and inelasticity. In: Angenheister, G. (Ed.), Physical Properties of Rocks, Landoldt-Börnstein, group V, Vol. 1, Subvol. b, Springer, Berlin, pp. 1-147.

Getting, I.C., 1998. The practical pressure scale: fixing fixed points and future prospects. Eos 79, F830.

Haussuehl, S., Wallrafen, F., Recker, K., Eckstein, J., 1980. Growth, elastic properties and phase transition of orthorhombic $\mathrm{Li}_{2} \mathrm{Ge}_{7} \mathrm{O}_{15}$. Z. Kristallogr. 153, 329-337.

Hearmon, R.F.S., 1984. The elastic constants of crystals and other anisotropic materials. In: Hellwege, K.H., Hellwege, A.M. (Eds), Landolt-Börnstein Tables, III/18, 559 p. Springer, Berlin, pp. 1-154.

Hirose, K., Fei, Y., Ono, S., Yagi, T., Funakoshi, K., 2001. In situ measurements of the phase transition boundary in $\mathrm{Mg}_{3} \mathrm{Al}_{2} \mathrm{Si}_{3} \mathrm{O}_{12}$ : implications for the nature of the seismic discontinuities in the Earth's mantle. Earth Planet. Sci. Lett. 184, 567-573.

Hoffbauer, W., Will, G., Lauterjung, J., 1985. Compressibility of forsterite up to $300 \mathrm{kbar}$ measured with synchrotron radiation. Z. Kristallogr. 170, 80-81, (in German).

Holland, T.J.B., Redfern, S.A.T., 1997. Unit cell refinement from powder diffraction data: the use of regression diagnostics. Mineral. Mag. 61, 65-77.

Kanzaki, M., 1991. Ortho/clinoenstatite transition. Phys. Chem. Miner. 17, 726-730.

Kern, H., 1982. Elastic-wave velocity in crustal and mantle rocks at high pressure and temperature: the role of the high-low quartz transition and of dehydration reactions. Phys. Earth Planet. Interiors 29, 12-23.

Knoche, R., Webb, S.L., Rubie, D.C., 1997. Experimental determination of acoustic wave velocities at Earth mantle condition using a multianvil press. Phys. Chem. Earth 22, 125-130.

Knoche, R., Webb, S.L., Rubie, D.C., 1998. Measurements of acoustic wave velocities at $P-T$ conditions of the Earth's mantle. In: Manghnani, M.H., Yagi, T. (Eds), Properties of Earth and Planetary Materials at High Pressure and Temperature, Geophysical Monograph 101. AGU, Washington, DC, pp. $119-128$.

Kohlstedt, D.L., Keppler, H., Rubie, D.C., 1996. Solubility of water in the $\alpha, \beta$ and $\gamma$ phases of $(\mathrm{Mg}, \mathrm{Fe})_{2} \mathrm{SiO}_{4}$. Contrib. Mineral. Petrol. 123, 345-357.

Kung, J., Gwanmesia, G.D., Liu, J., Li, B., Liebermann, R.C., 2000. PV3T experiments: simultaneous measurement of sound velocities $\left(V_{\mathrm{p}}\right.$ and $\left.V_{\mathrm{s}}\right)$ and sample volume $(V)$ of polycrystalline specimens of mantle minerals at high pressures $(P)$ and temperatures $(T)$. Eos 81 (No. 48), F1151.

Kung, J., Angel, R.J., Ross, N.L., 2001a. Elasticity of $\mathrm{CaSnO}_{3}$ perovskite. Phys. Chem. Miner. $28,35-43$. Kung, J., Weidner, D.J., Li, B., Liebermann, R.C., 2001b. Determination of the elastic properties at high pressure without pressure scale. Eos 82, F1383. 
Kung, J., Li, B., Weidner, D.J., Zhang, J., Liebermann, R.C., 2002. Elasticity of (Mg0.83 Fe0.17)O ferropericlase at high pressure: ultrasonic measurements in conjunction with X-radiation techniques. Earth Planet. Sci. Lett. 203, 557-566.

Kung, J., Li, B., Uchida, T., Wang, Y., Neuville, D., Liebermann, R.C., 2004. In situ measurements of sound velocities and densities across the orthopyroxene - high pressure clinopyroxene transition in $\mathrm{MgSiO}_{3}$ at high pressure. Phys. Earth Planet. Interiors 147, $27-44$.

Lauterjung, J., Will, G., 1985. Investigation of olivine-spinel phase transition with time-delayed energydispersive X-ray diffraction using the example of $\mathrm{Mg}_{2} \mathrm{GeO}_{4}$. Z. Kristallogr. 170, 117-119, (in German).

$\mathrm{Li}, \mathrm{B}$., 2003. Compressional and shear wave velocities of ringwoodite $\gamma-\mathrm{Mg}_{2} \mathrm{SiO}_{4}$ to $12 \mathrm{GPa}$. Am. Mineral. $88,1312-1317$.

Li, B., Gwanmesia, G.D., Liebermann, R.C., 1996a. Sound velocities of olivine and beta polymorphs of $\mathrm{Mg}_{2} \mathrm{SiO}_{4}$ at Earth's transition zone pressures. Geophys. Res. Lett. 23 (No. 17), 2259-2262.

Li, B., Jackson, I., Gasparik, T., Liebermann, R.C., 1996b. Elastic wave velocity measurement in multianvil apparatus to $10 \mathrm{GPa}$ using ultrasonic interferometry. Phys. Earth Planet. Interiors 98, 79-91.

Li, B., Chen, G., Gwanmesia, G.D., Liebermann, R.C., 1998. Sound velocity measurements at mantle transition zone conditions of pressure and temperature using ultrasonic interferometry in a multianvil apparatus. In: Manghnani, M.H., Yagi, T. (Eds), Properties of Earth and Planetary Materials at High Pressure and Temperature, Geophysical Monograph 101. AGU, Washington, DC, pp. $41-61$.

Q7 Li, X., Kind, R., Priestley, K., Sobolev, S.V., Tilmann, F., Yuan, X., Weber, M., 2000. Mapping the Hawaiian plume conduit with converted seismic waves. Nature 405, 938-941.

Li, B., Liebermann, R.C., Weidner, D.J., 2001a. $P-V-V_{p}-V_{s}-T$ measurement on wadsleyite to $7 \mathrm{GPa}$ and $873 \mathrm{~K}$ : implications for the 410-km seismic discontinuity. J. Geophys. Res. 106 (B12), 30575-30591.

Li, B., Vaughan, M.T., Kung, J., Weidner, D.J., 2001b. Direct length measurement using X-radiography for the determination of acoustic velocities at high pressure and high temperature. NSLS Activity Report 2, 103-106.

Li, B., Chen, K., Kung, J., Liebermann, R.C., Weidner, D.J., 2002. Sound velocity measurement using transfer function method. J. Phys. Condens. Matter 14, 11337-11342.

Liebermann, R.C., Li, B., 1998. Elasticity at high pressure and temperatures. In: Hemley, R. (Ed.), Ultrahigh-Pressure Mineralogy, Rev. Mineral., Vol. 37, pp. 459-492.

Q7 Liebermann, R.C., Ringwood, A.E., 1976. Elastic properties of anorthite and the nature of the lunar crust. Earth Planet. Sci. Lett. 31, 69-74.

McSkimin, H.J., 1950. Ultrasonic measurement techniques applicable to small solid specimens. J. Acoust. Soc. Am. 22, 413-418.

Mueller, H.J., Lauterjung, J., Schilling, F.R., Lathe, C., Nover, G., 2002. Symmetric and asymmetric interferometric method for ultrasonic compressional and shear wave velocity measurements in pistoncylinder and multi-anvil high-pressure apparatus. Eur. J. Mineral. 14, 581-589.

Mueller, H.J., Schilling, F.R., Lauterjung, J., Lathe, C., 2003. A standard free pressure calibration using simultaneous XRD and elastic property measurements in a multi-anvil device. Eur. J. Mineral. 15, $865-873$.

Niesler, H., Jackson, I., 1989. Pressure derivatives of elastic wave velocities from ultrasonic interferometric measurements on jacketed polycrystals. J. Acoust. Soc. Am. 86, 1573-1585.

Nishiyama, N., Yagi, T., 2003. Phase relation and mineral chemistry in pyrolite to $2200^{\circ} \mathrm{C}$ under the lower mantle pressures and implications for dynamics of mantle plumes. J. Geophys. Res. 108, 7-1-7-12.

Oguri, K., Funamori, N., Uchida, T., Miyajima, N., Yagi, T., Fujino, K., 2000. Post-garnet transition in the natural pyrope: a multi-anvil study based on in situ X-ray diffraction and transmission electron microscopy. Phys. Earth Planet. Interiors 122, 175-186.

Ohashi, Y., 1984. Polysynthetically-twinned structures of enstatite and wollastonite. Phys. Chem. Miner. 10, 217-229.

Poirier, J.P., 1982. On transformation plasticity. J. Geophys. Res. 87, 6791-6797.

Rapppaport, T.S., 2002. Wireless communications. Principles and Practice. 2/2. Chap. 6. Modulation Techniques for Mobile Radio. [online] [Cited 2002 Pearson]. Available from: http://www.utexas.edu/ $\sim$ wireless/EE381K11_Spring03/Presentation_6.ppt.

Rigden, S.M., Jackson, I., Niesler, H., Liebermann, R.C., Ringwood, A.E., 1988. Pressure dependence of the elastic wave velocities for $\mathrm{Mg}_{2} \mathrm{GeO}_{4}$ spinel up to $3 \mathrm{GPa}$. Geophys. Res. Lett. 15, 604-608. 
Rigden, S.M., Gwanmesia, G.D., Jackson, I., Liebermann, R.C., 1992. Progress in high-pressure ultrasonic interferometry, the pressure dependence of elasticity of $\mathrm{Mg}_{2} \mathrm{SiO}_{4}$ polymorphs and constraints on the composition of the transition zone of the Earth's mantle. In: Syono, Y., Manghnani, M. (Eds), High Pressure Research: Application to Earth and Planetary Sciences. Terra Scientific Publishing Co. and AGU, Tokyo and Washington, DC, pp. 167-182.

Rigden, S.M., Gwanmesia, D., Liebermann, R.C., 1994. Elastic wave velocities of a pyrope-majorite garnet to $3 \mathrm{GPa}$. Phys. Earth Planet. Interiors 86, 35-44.

Rybacki, E., Dresen, G., 2000. Dislocation and diffusion creep of synthetic anorthite aggregates. J. Geophys. Res. 105 (B11), 26017-26036.

Rybacki, E., Dresen, D.H., Wirth, R., 1998. Creep of synthetic anorthosite at high temperature and high pressure. Eos 79, F852.

Schilling, F.R., 1998. PETROPHYSIK - Ein mineralogischer Ansatz, Habilitationsschrift, Fachbereich Geowissenschaften, Freie Universität Berlin, p. 259, (in German).

Schmidt, C., Bruhn, D., Wirth, R., 2002. Experimental evidence of transformation plasticity in silicates: minimum of creep strength in quartz. Earth Planet. Sci. Lett. 6468, 1-8.

Schreiber, E., Anderson, O.L., Soga, N., 1973. Elastic Constants and their Measurement. McGraw-Hill Book Company, New York, p. 196.

Seidel, H-P., Myszkowski, K., 2004. Computer graphics - signal processing. [online] Portable Document Format. Available from: http://mpi-sb.mpg.de/units/ag4/teaching/uebung/lecture23.pdf.

Shen, A.H., Reichmann, H.-J., Chen, G., Angel, R.J., Bassett, W.A., Spetzler, H., 1998. GHz ultrasonic interferometry in a diamond anvil cell: P-wave velocities in periclase to $4.4 \mathrm{GPa}$ and $207^{\circ} \mathrm{C}$. In: Manghnani, M.H., Yagi, T. (Eds), Properties of Earth and Planetary Materials at High Pressure and Temperature, Geophysical Monograph 101. AGU, Washington, DC, pp. 71-77.

Shimomura, O., Yamaoka, S., Yagi, T., Wakutsuki, M., Tsuji, K., Kawamura, H., Hamaya, N., Fukunaga, O., Aoki, K., Akimoto, S., 1985. Multi-anvil type X-ray system for synchrotron radiation. In: Minomura, S. (Ed.), Solid State Physics Under Pressure. Terra Science Publishing, Tokyo, pp. $351-356$.

Shinmei, T., Tomioka, N., Fujino, K., Kuroda, K., Irifune, T., 1999. In situ X-ray diffraction study of enstatite up to $12 \mathrm{GPa}$ and $1473 \mathrm{~K}$ and equations of state. Am. Mineral. 84, 1588-1994.

Spetzler, H., Yoneda, A., 1993. Performance of the complete travel-time equation of state at simultaneous high pressure and temperature. Pure Appl. Geophys. 141, 379-392.

Suzuki, T., Akaogi, M., Yagi, T., 1996. Pressure dependence of Ni, Co and Mn partitioning between iron hydride and olivine, magnesiowüstite and pyroxene. Phys. Earth Planet. Interiors 96, 209-220.

Ulmer, P., Stalder, R., 2001. The $\mathrm{Mg}(\mathrm{Fe}) \mathrm{SiO}_{3}$ orthoenstatite-clinoenstatite transitions at high pressures and temperatures determined by Raman-spectroscopy on quenched samples. Am. Mineral. 86, $1267-1274$.

van der Hilst, R., 1995. Complex morphology of subducted lithosphere in the mantle beneath the Tonga trench. Nature 374, 154-157.

Vaughan, M.T., 1993. In situ X-ray diffraction using synchrotron radiation at high $P$ and $T$ in multianvil device. In: Luth, R.W. (Ed.), Experiments at High Pressure and Applications to the Earth's Mantle, Short Course Handbook 21. Mineralogical Association of Canada, Edmonton, Alberta, pp. $95-130$.

Vaughan, M.T., Weidner, D.J., Wang, J.H., Chen, J.H., Koleda, C.C., Getting, I.C., 1995. T-CUP: a new high-pressure apparatus for X-ray studies. NSL Activity Report, p. B140, Available from: http://www. chipr.sunysb.edu/sam85/tcup/tcup.html.

Webb, S.L., 1989. The elasticity of the upper mantle orthosilicates olivine and garnet to 3 GPa. Phys. Chem. Miner. 16, 684-692.

Will, G., Hinze, E., Nuding, W., 1982. Energy-dispersive X-ray diffraction applied to the study of minerals under pressure up to 200 kbar. In: Schreyer, W. (Ed.), High-Pressure Researches in Geoscience. Schweizerbart'sche Verlagsbuchhandlung, Stuttgart, pp. 177-201.

Wirth, R., 1996. Thin amorphous films $(1-3 \mathrm{~nm})$ at olivine grain boundaries in mantle xenoliths from San Carlos, Arizona. Contrib. Mineral. Pertol. 124, 44-54.

Yagi, T., 1988. MAX80: large-volume high-pressure apparatus combined with synchrotron radiation. Eos 69 (12), 18-27.

Yoneda, A., Spetzler, H., 1994. Temperature fluctuation and thermodynamic properties in Earth's lower mantle: an application of the complete travel time equation of state. Earth Planet. Sci. Lett. 126 (4), $369-377$. 
Zaug, J.M., Abramson, E.H., Brown, J.M., Slutsky, L.J., 1993. Sound velocities in olivine at Earth mantle pressures. Science 260, 1487-1489.

Zha, C.-S., Mao, H.K., Hemley, R.J., 2000. Elasticity of $\mathrm{MgO}$ and a primary pressure scale to $55 \mathrm{GPa}$. Proc. Natl Acad. Sci. 97, 13494-13499.

Zinn, P., Hinze, E., Lauterjung, J., Wirth, R., 1997. Kinetic and microstructural studies of the quartzcoesite phase transition. Phys. Chem. Earth 22, 105-111. 